\title{
The effect of Wolbachia on gene expression in Drosophila paulistorum and its implications for symbiont-induced host speciation
}

\author{
Guilherme C. Baião ${ }^{1}$, Daniela I. Schneider ${ }^{2,3}$, Wolfgang J. Miller ${ }^{2}$ and Lisa Klasson ${ }^{1 *}$ (D)
}

\begin{abstract}
Background: The Neotropical fruit fly Drosophila paulistorum (Diptera: Drosophilidae) is a species complex in statu nascendi comprising six reproductively isolated semispecies, each harboring mutualistic Wolbachia strains. Although wild type flies of each semispecies are isolated from the others by both pre- and postmating incompatibilities, mating between semispecies and successful offspring development can be achieved once flies are treated with antibiotics to reduce Wolbachia titer. Here we use RNA-seq to study the impact of Wolbachia on D. paulistorum and investigate the hypothesis that the symbiont may play a role in host speciation. For that goal, we analyze samples of heads and abdomens of both sexes of the Amazonian, Centro American and Orinocan semispecies of D. paulistorum.
\end{abstract}

Results: We identify between 175 and 1192 differentially expressed genes associated with a variety of biological processes that respond either globally or according to tissue, sex or condition in the three semispecies. Some of the functions associated with differentially expressed genes are known to be affected by Wolbachia in other species, such as metabolism and immunity, whereas others represent putative novel phenotypes involving muscular functions, pheromone signaling, and visual perception.

Conclusions: Our results show that Wolbachia affect a large number of biological functions in D. paulistorum, particularly when present in high titer. We suggest that the significant metabolic impact of the infection on the host may cause several of the other putative and observed phenotypes. We also speculate that the observed differential expression of genes associated with chemical communication and reproduction may be associated with the emergence of pre- and postmating barriers between semispecies, which supports a role for Wolbachia in the speciation of D. paulistorum.

Keywords: Speciation, symbiosis, Wolbachia, transcriptome, Drosophila paulistorum, host-symbiont interactions

\section{Background}

Speciation is still one of the overarching concepts in biology. The process is usually assumed to involve the development of both pre- and postmating isolation, and a large number of studies have been dedicated to understanding how these arise [1]. One factor which has been gaining attention as a contributor to the speciation

\footnotetext{
* Correspondence: lisa.klasson@icm.uu.se

${ }^{1}$ Molecular evolution, Department of Cell and Molecular Biology, Uppsala

University, Husargatan 3, 75124 Uppsala, Sweden

Full list of author information is available at the end of the article
}

process in animals is the influence of microbial symbionts [2]. Growing evidence indicates that microorganisms affect host traits, such as behavior, metabolism, immunity and reproduction, which in turn can influence mating incompatibilities [3]. Insects, in particular, are associated with a large variety of microbial symbionts that are often implicated as contributors to the remarkable species diversity in this group of organisms [2].

The Neotropical fruit fly Drosophila paulistorum (Diptera: Drosophilidae) is considered a classical example of incipient speciation. Its six semispecies, Amazonian

(c) The Author(s). 2019 Open Access This article is distributed under the terms of the Creative Commons Attribution 4.0 International License (http://creativecommons.org/licenses/by/4.0/), which permits unrestricted use, distribution, and reproduction in any medium, provided you give appropriate credit to the original author(s) and the source, provide a link to the Creative Commons license, and indicate if changes were made. The Creative Commons Public Domain Dedication waiver (http://creativecommons.org/publicdomain/zero/1.0/) applies to the data made available in this article, unless otherwise stated. 
(AM), Andean-Brazilian (AB), Centro American (CA), Interior (IN), Orinocan (OR) and Transitional (TR) [4, 5] are morphologically similar, have partially overlapping geographical distributions, and yet show both pre- and postmating barriers to hybridization [6, 7]. Premating isolation is observed through female rejection of males belonging to other semispecies $[7,8]$, while postmating barriers manifest as embryonic lethality and male sterility in the rare hybrids that develop into adults $[9,10]$. Early studies suggested that the reproductive incompatibility observed in D. paulistorum was due to a microbial infection $[11,12]$, but it was only recently determined that the microbe in question is Wolbachia [8].

Wolbachia (Alphaproteobacteria) are widespread endosymbionts of invertebrates, infecting over $60 \%$ of insect species [13] as well as Arachnids [14], Crustaceans [15] and Nematodes [16]. They are vertically transmitted through the maternal line and infect primarily the reproductive tissues, although other organs will often also host bacteria [17, 18]. Wolbachia have been found to participate in a range of biological interactions with arthropod hosts, from nutritional mutualism and protection against pathogens to various forms of reproductive parasitism [19, 20].

Wolbachia have high prevalence among arthropods, but they are often facultative for these hosts. However, in D. paulistorum, Wolbachia are obligate mutualists necessary for proper gonad development [8], analogous to what is observed in some wasps of the genus Asobara $[21,22]$. The mutualistic nature of Wolbachia is further supported by its presence in every $D$. paulistorum semispecies tested so far, although the titer of the infection can vary from high to only a few endosymbiont cells per fly [8]. In such low titer cases, Wolbachia presence is below the detection limit of a standard PCR, and more sensitive techniques must be used [8]. Remarkably, even very low titer infections are capable of inducing reproductive incompatibility, as successful mating across semispecies is facilitated once the Wolbachia titer is reduced through mild antibiotic treatment $[8,11]$. Specifically, antibiotic treated females become more accepting of males belonging to other semispecies [8] and hybrid male sterility is partially rescued after treatment of the parents [11]. This suggests that, in this system, the endosymbiont is able to prevent hybridization by inducing not only postmating incompatibility but also premating isolation between semispecies.

Little is known about the influence of Wolbachia on biological functions of $D$. paulistorum, but a recent study shows that the symbiont affects male pheromone profiles and thereby modulates mate recognition in that species [23]. This suggests the effect of Wolbachia on premating isolation might be associated with changes in host chemical communication
[8]. Wolbachia has been shown to infect brain regions responsible for sensory perception in $D$. paulistorum [17], and many of the 50 odorant-binding proteins (OBPs) encoded by the Drosophila genome could be targets for affecting reception of chemical stimuli [24, 25]. An important group of pheromones in Drosophila are the cuticle hydrocarbons (CHCs), molecules derived from fatty acid metabolism [26, 27]. Unique $\mathrm{CHC}$ profiles have been associated with each semispecies and sex of D. paulistorum [28], and in vivo tests demonstrated that cuticular extracts from one semispecies can inhibit courtship by males of others, supporting their role in semispecies isolation [28]. Consequently, Wolbachia manipulation of genes related to $\mathrm{CHC}$ production and/or perception could affect host premating behavior.

The influence of Wolbachia on host postmating compatibility is usually associated with cytoplasmic incompatibility (CI). CI is the most commonly observed Wolbachia-induced host manipulation and is characterized by partial or complete embryonic lethality in crosses between infected males and non-infected females or between hosts carrying incompatible symbiont strains. It is not known whether $\mathrm{CI}$ has a role in the incompatibilities between $D$. paulistorum semispecies, but the phenotype has been suggested as a driver of speciation in other systems due to its potential to reproductively isolate insect populations $[8,29,30]$. On a cellular level, CI affects paternal chromosome condensation during the first embryonic mitosis, leading to lethal chromatin missegregation in anaphase $[31,32]$. Recent studies have also elucidated some of the Wolbachia proteins responsible for inducing $\mathrm{CI}$ in $D$. melanogaster $[31,33$, but very little is known about which host genes are involved in the process.

In the present study, we use RNA-seq to investigate the impact of Wolbachia on the biology of three semispecies of Drosophila paulistorum, two with low titer Wolbachia infections, AM and CA, and one with a high titer Wolbachia infection, OR. For each semispecies, we analyze samples from heads and abdomens of both sexes from wild type (WT) flies as well as from corresponding antibiotic-treated and gut flora restored (GFR) individuals. Our goal is to get a better understanding of how Wolbachia affects its host and to investigate whether this interaction contributes to the speciation process of D. paulistorum. We find that Wolbachia affects hundreds of genes associated with global and conditionspecific biological processes, including metabolism, immunity, olfactory perception, vision and reproduction. We suggest that the metabolic changes caused by $\mathrm{Wol}$ bachia might be responsible for other observed phenotypes and discuss the possibility that some of the affected genes and processes support a role for Wolbachia in the speciation of $D$. paulistorum. 


\section{Results}

Data, transcriptome assembly and quality assessment RNA-seq data was collected from heads and abdomens of female and male WT and GFR treated flies of the AM, CA and OR semispecies. While WT flies contain the natural Wolbachia titer, GFR flies were subjected to mild antibiotic treatment in order to reduce the Wolbachia titer. To avoid host effects stemming from removal of gut microbes, the GFR flies had their gut flora restored after antibiotic treatment (see Materials and Methods). The effectivity of the antibiotic treatment to reduce Wolbachia titer in both low and high titer Wolbachia in D. paulistorum has been previously tested with qPCR [23] (unpublished data). Additionally, we see a strong reduction in the number of reads mapping to Wolbachia in samples from GFR compared to WT flies of the high titer semispecies OR (Additional file 1). The absence of differentially expressed (DE) non-Wolbachia bacterial genes between WT and GFR samples in all but one condition (data not shown) indicates that gut microbes likely have a very small impact on the results.

Following read quality control, the transcriptomes for the AM, CA and OR semispecies of D. paulistorum were assembled separately with Trinity using reads from most samples of that semispecies (see Materials and Methods) . Before further analyses, each assembly was then filtered to reduce sequence redundancy and remove non-coding contigs. Additionally, contigs with multiple open reading frames (ORFs) were split. The final reference assemblies showed very high completeness, as measured by recovery of BUSCO markers, and contained between 3300036000 ORFs each (Table 1). Most ORFs were of Drosophila origin, with a minority associated to either bacteria or yeast (Table 1), and the three assemblies show very high overlap in Drosophila gene content (Additional file 2: Figure S1). The OR transcriptome was the only one containing Wolbachia ORFs (1.43\%), which is consistent with OR WT samples having considerably more Wolbachia reads than any other condition (Table 1, Additional file 1) and with OR being the only semispecies with a high titer Wolbachia infection.

The reads from each semispecies were mapped to their respective reference transcriptome assembly and counted using FeatureCounts followed by principal component analysis (PCA) in DEseq2. Gene expression in each semispecies varied according to sex, tissue and Wolbachia infection, although the latter on a smaller scale (Fig. 1, Additional file 2: Figures S2, S3). Expression differences between sexes were greater in abdomens than in heads, and particularly distinct between male abdomens and other conditions (Fig. 1, Additional file 2: Figures S2, S3). Variation between biological replicates was low (Fig. 1).
Table 1 Assembly metrics for the transcriptomes used in the differential expression analysis of each semispecies

\begin{tabular}{|c|c|c|c|}
\hline & AM & CA & OR \\
\hline Number of contigs (ORFs) & 35233 & 36422 & 33680 \\
\hline Maximum contig length & 26253 & 27348 & 23729 \\
\hline Average contig length & 1384 & 1284 & 1374 \\
\hline Contig N50 & 1923 & 1781 & 1889 \\
\hline \multicolumn{4}{|l|}{ BUSCO marker recovery (\%) } \\
\hline Arthropod & 98.50 & 98.50 & 98.78 \\
\hline Insecta & 98.01 & 98.13 & 98.37 \\
\hline Diptera & 95.68 & 95.61 & 95.93 \\
\hline \multicolumn{4}{|l|}{ ORF completeness (\%) } \\
\hline Complete & 41.82 & 37.32 & 41.20 \\
\hline 3-prime partial & 12.74 & 13.68 & 13.23 \\
\hline 5-prime partial & 23.13 & 23.07 & 23.41 \\
\hline Internal & 22.31 & 25.93 & 22.16 \\
\hline \multicolumn{4}{|l|}{ ORFs associated to: (\%) } \\
\hline Drosophila & 77.94 & 77.28 & 82.92 \\
\hline Wolbachia & 0.00 & 0.00 & 1.43 \\
\hline Other bacteria & 6.36 & 10.10 & 3.33 \\
\hline Yeast & 1.18 & 0.98 & 0.96 \\
\hline Not assigned ${ }^{\mathrm{a}}$ & 14.51 & 11.64 & 11.35 \\
\hline
\end{tabular}

\section{Differential expression analysis in the three semispecies}

Separate differential expression analyses were done for each sex and tissue in each semispecies using DESeq2 and an adjusted p-value of 0.05 as significance cutoff. GFR was set as reference condition, which means that the direction of gene expression change is due to Wolbachia rather than antibiotic treatment. Since our focus is on investigating the effect of Wolbachia on the host gene expression, all results, numbers, figures and discussion presented from here on refer to Drosophila genes only, unless otherwise noted.

A total of 175, 209 and 1192 Drosophila genes were differentially expressed between WT and GFR flies in AM, CA and OR, respectively. Out of these, 67-81\% could be assigned putative functional annotations (Table 2). A heatmap of the DE genes in OR (Fig. 1b) allows visualization of the expression differences between WT and GFR, which clearly are mild compared to differences between tissues and sex. A complete list of DE genes in the three semispecies and their respective annotations is available in Additional files 3, 4 and 5. We identified one up- and 9 downregulated genes which are DE in all three semispecies, irrespectively of condition (Fig. 2). Among these we find $R y R$, a calcium channel which is important for muscle contraction, FASN2, which is 

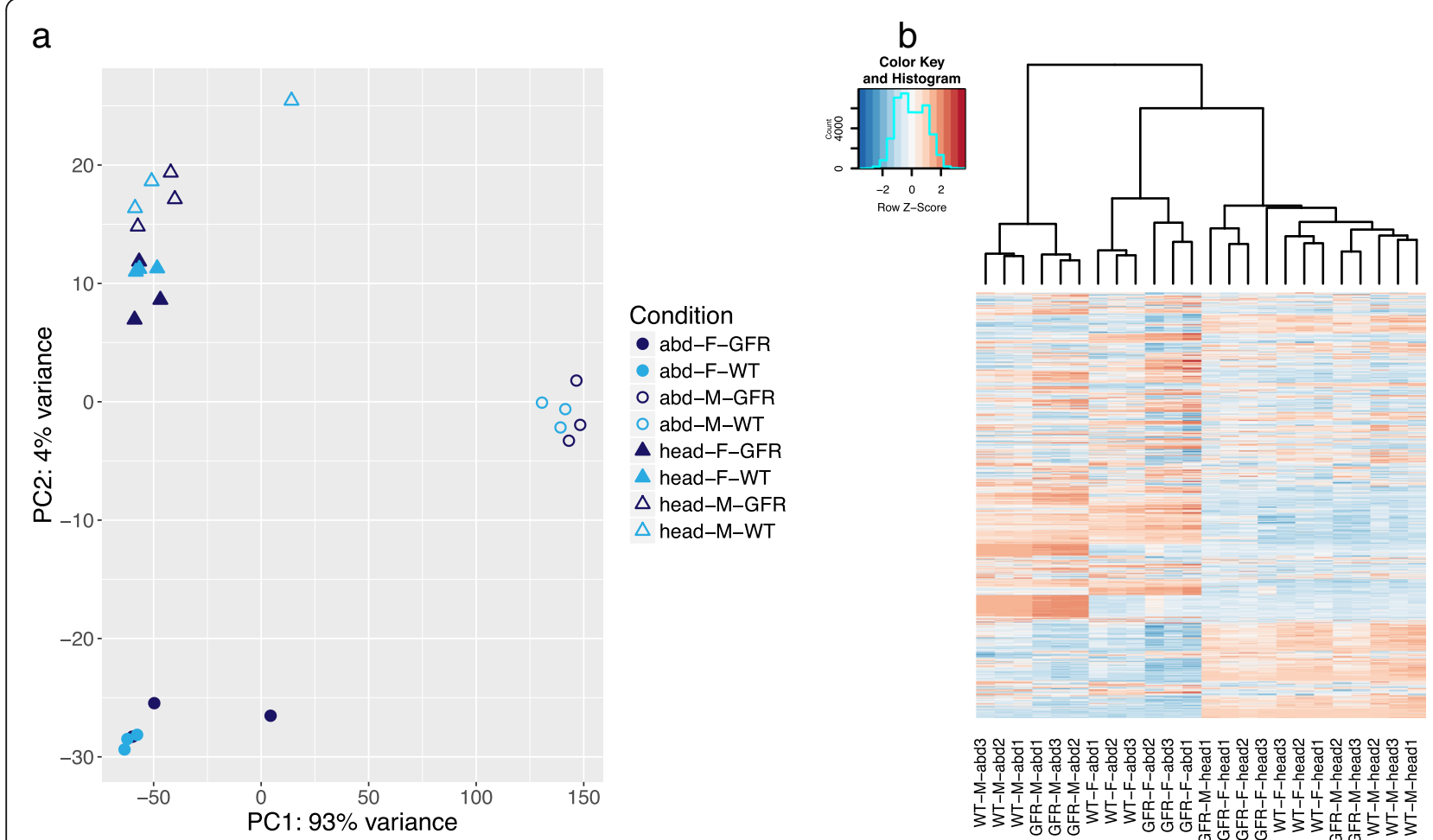

Fig. 1 Principal component analysis (a) and heatmap (b) of expression data of the OR semispecies. The PCA is based on all Drosophila genes in the analysis, while the heatmap shows only DE genes. F: female, M: male, WT: wild type, GFR: gut flora restored, abd: abdomen

involved in fatty acid metabolism and implicated in Drosophila speciation (see Discussion), the cytochrome $\mathrm{P} 450$ gene Cyp6g1, and several uncharacterized genes (Additional file 6). On the other hand, a small number of DE genes were present only in one of the assemblies, 4, 2 and 6 genes in AM, CA and OR respectively. None of these were annotated (Additional file 2: Figure S1).

Most DE genes in the CA and OR semispecies are downregulated in WT (Table 2), and these are proportionally better annotated than upregulated genes in both semispecies. The opposite is seen in AM, where DE genes are more commonly upregulated in WT and these are generally better annotated. (Table 2). In OR, abdomens have more than twice as many $\mathrm{DE}$ genes than heads (Table 2). A similar trend is observed in CA, but again the opposite is found in AM (Table 2).

The lower number of DE genes in AM and CA compared to OR (Table 2) might be a consequence of differences in infection titer or Wolbachia strains between the semispecies (see Discussion). Given these reduced numbers of DE genes in the AM and CA, from here on we will only present the results from the $\mathrm{DE}$ analysis of the OR semispecies, unless otherwise stated.

Table 2 Number of DE Drosophila genes in the three semispecies

\begin{tabular}{|c|c|c|c|c|}
\hline Condition & Regulation $^{a}$ & AM (annotated) & CA (annotated) & OR (annotated) \\
\hline \multirow[t]{2}{*}{ Female abdomen } & Up & $7(7)$ & $3(2)$ & $325(203)$ \\
\hline & Down & $24(18)$ & $104(88)$ & $164(143)$ \\
\hline \multirow[t]{2}{*}{ Female head } & Up & $59(50)$ & $18(14)$ & $36(23)$ \\
\hline & Down & $23(17)$ & $30(25)$ & $202(188)$ \\
\hline \multirow[t]{2}{*}{ Male abdomen } & Up & $37(20)$ & $7(4)$ & $223(108)$ \\
\hline & Down & $7(5)$ & $15(14)$ & $324(282)$ \\
\hline \multirow[t]{2}{*}{ Male head } & Up & $20(17)$ & $3(2)$ & $25(8)$ \\
\hline & Down & $55(21)$ & $13(7)$ & $225(203)$ \\
\hline Total unique DE genes & & $175(139)$ & $209(142)$ & $1192(921)$ \\
\hline
\end{tabular}

\footnotetext{
${ }^{a}$ Up- and downregulation presented as a response to Wolbachia infection
} 


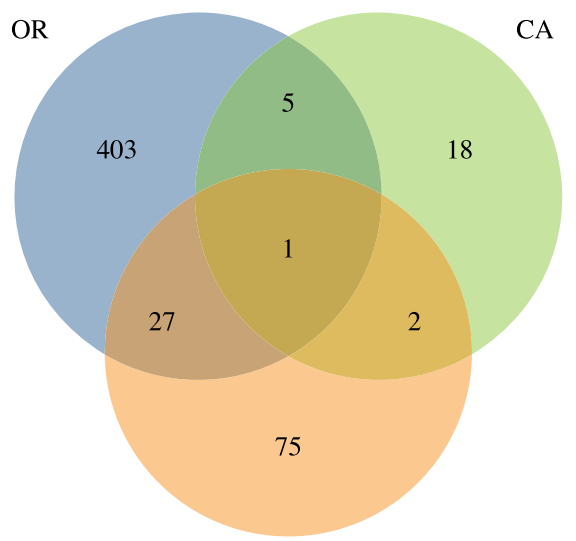

$\mathrm{AM}$

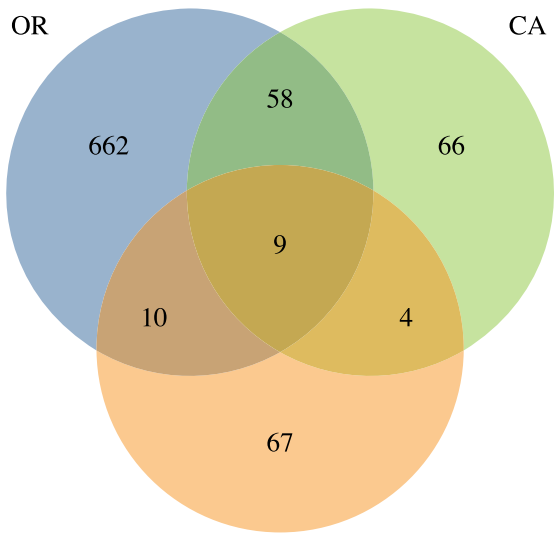

$\mathrm{AM}$

Fig. 2 Number of genes up- (a) or downregulated (b) in one or multiple semispecies. Shared genes are identified as those included in the same OrthoMCL cluster. All conditions are pooled in this analysis and GFR is used as the reference

Patterns of differential expression in the OR semispecies Most DE genes in the three semispecies are DE in a single tissue, sex or condition (i.e. the combination of tissue and sex), with only a small number DE in both sexes or tissues (Fig. 3, Additional file 2: Figures S4, S5). In the OR semispecies, tissue-specific responses include several upregulated genes in both male and female WT abdomens as well as genes downregulated in both male and female WT heads (Fig. 3). Sex-specific responses to Wolbachia are less common and more prominent in males, in which 47 genes are downregulated in both abdomens and heads (Fig. 3).

Male abdomens have the largest number of DE genes (Table 2) and female abdomens the highest condition-

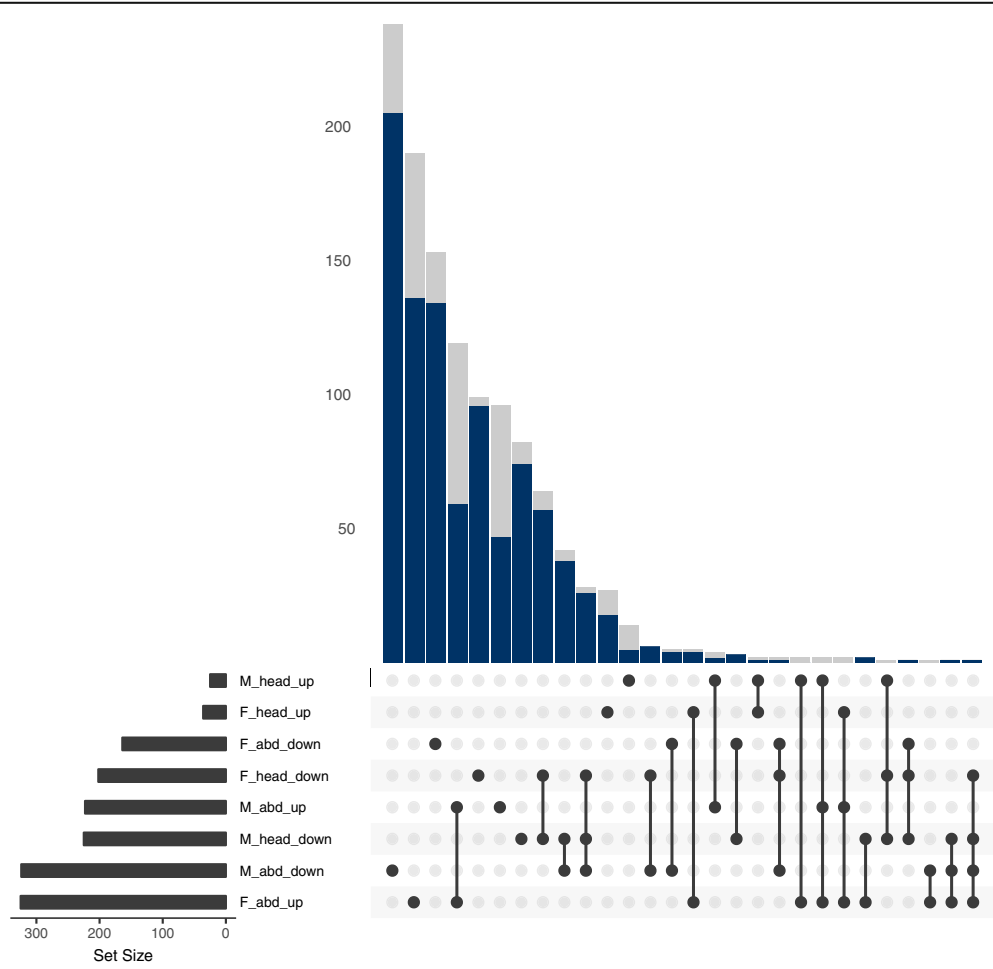

Fig. 3 Number of genes differentially expressed in one or multiple conditions of the OR semispecies. A black dot indicates the presence of DE genes for the condition named on the left side. Dots linked by lines represent DE genes in multiple conditions. Vertical bars above the dots correspond to the number of annotated (blue) and unannotated (grey) DE genes present in the condition(s) marked with a dot. Horizontal black bars on the lower left indicate how many genes are DE in each condition. F: female, M: male, WT: wild type, GFR: gut flora restored, abd: abdomen, up: upregulated, down: downregulated 
specificity, with $93 \%$ of the down- and $58 \%$ of the upregulated genes being exclusive to it (Fig. 3). In heads, males have only a slightly larger number of $\mathrm{DE}$ genes than females, but again females have a higher conditionspecificity (Table 2, Fig. 3 ).

\section{Enrichment of biological process among DE genes in the OR semispecies}

Using TopGO and GO term annotation, we analyzed which biological functions were enriched among the $\mathrm{DE}$ genes identified in each condition (Table 3, Additional file 7). We found that the DE genes between WT and GFR participate in a wide range of biological processes and are enriched either globally or in specific tissues and conditions, indicating that responses to Wolbachia can be either general or localized.

In the following sections, we present the main biological functions associated with the DE genes in our dataset based on GO term enrichment, pathway analyses and manual curation (Table 4). For each function, we mention if the response is global or specific and highlight DE genes with high fold change, as these are likely the most reliable and biologically relevant signals in the analysis (Additional files 3, 4 and 5). DE genes with high fold change are those having a fold change higher than at least one standard deviation from the mean fold change of the condition.

\section{Metabolism}

A large number of genes involved in carbohydrate, fatty acid and amino acid metabolism are DE in multiple conditions, often with high fold changes (Additional file 3). Most of these are downregulated in WT flies, particularly in female and male heads and in the male abdomens (Table 4).

The precise identities of the affected genes differ somewhat between conditions, even so, a relatively clear pattern exists for up- and down-regulated genes in glucose and energy metabolism (Fig. 4). In WT flies, there is an increased expression of genes involved in the TCA cycle (abdomens), whereas in GFR flies there is an increased expression of genes involved in the pentose phosphate pathway, the breakdown of glycogen to UDP-glucose (males) and beta-oxidation of fatty acids (female heads). This pattern suggests increased catabolism in WT flies, possibly with an increased flux through the TCA cycle, whereas GFR flies have more anabolic metabolism and use beta-oxidation for producing acetyl-CoA as well as the pentose phosphate pathway for generating precursors for nucleotide, amino acid or lipid biosynthesis.

\section{Lipids}

The putative increased anabolism in GFR flies is supported by the upregulation of genes involved in fatty acid biosynthesis and phospholipid metabolism in multiple conditions (Table 4). These include several genes in pathways for converting glycerate to phosphatidic acid, phosphatic acid to phosphatidylethanolamine and phosphatidylcholine, and ethanolamine to phosphoethanolamine.

Furthermore, GFR flies have a higher expression of five genes putatively involved in cholesterol hydrolysis (CG8093, CG31091, CG2772, CG7329 and CG18302) as well as three genes containing the Niemann-Pick type C-2 domain, which has a potential role in intracellular cholesterol transport.

\section{Nucleotides}

Several genes related to purine biosynthesis are upregulated in the head of female GFR flies, which further supports their increased anabolism. The presence of genes for de novo purine synthesis in Wolbachia genomes indicates that the symbiont is likely able to synthesize such molecules, and the increased expression of purine biosynthesis genes in GFR flies might thus be a compensation for the loss of Wolbachia-provided purines. In filarial nematodes, where Wolbachia is also an obligate mutualist, it has been suggested that one of its functions is to provide purines for the host [34].

\section{Amino acids}

Although Wolbachia relies on the host for obtaining most amino acids [35], we only observe one amino acid biosynthesis gene with a higher expression in WT flies (glutamine synthetase 2). Hence, increased host biosynthesis is likely not a source of amino acids for Wolbachia. However, and again in agreement with the putative increased anabolism in GFR flies, some amino acid biosynthetic genes have lower expression in WT flies. These include two genes in the pathway converting glutamate to proline and several genes involved in serine and glycine metabolism (Table 4, Fig. 4), all with lower expression in male and/or female heads. Collectively, this suggests that both glycine and serine levels might be reduced in the heads of WT flies.

Furthermore, two putative components of the glycine cleavage system are downregulated in heads of WT males and females. This is a further indication that the level of glycine is lower in WT flies, as the activity of this system is normally regulated by the concentration of glycine. Apart from its role in glycine breakdown, the glycine cleavage system uses tetrahydrofolate (THF) to generate 5,10-Methylene-THF, which can be further utilized in purine biosynthesis or as a substrate for the enzyme serine hydroxymethyl transferase. Several additional genes involved in THF conversions, such as pug and CG34424, or in purine biosynthesis, like Nmdmc and CG11089, also have a lower expression in heads of WT flies. An increase in THF conversion is a 
Table 3 Ten most significantly enriched GO terms in each condition of the OR semispecies

\begin{tabular}{|c|c|c|c|c|c|}
\hline GO Term & Annotation & $\begin{array}{l}\mathrm{Nr} \\
\text { Ann. }\end{array}$ & $\begin{array}{l}\mathrm{Nr} \\
\mathrm{DE}^{\mathrm{a}}\end{array}$ & $\begin{array}{l}\mathrm{Nr} \\
\text { Exp. }^{a}\end{array}$ & Signif." \\
\hline \multicolumn{6}{|c|}{ Female abdomen upregulated } \\
\hline GO:0045214 & sarcomere organization & 27 & 12 & 0.58 & $9.20 \mathrm{E}-14$ \\
\hline GO:0030239 & myofibril assembly & 38 & 18 & 0.82 & 4.90E-09 \\
\hline GO:0007498 & mesoderm development & 74 & 12 & 1.6 & 4.80E-08 \\
\hline GO:0006936 & muscle contraction & 23 & 10 & 0.5 & $4.50 \mathrm{E}-07$ \\
\hline GO:0071688 & striated muscle myosin thick filament assembly & 5 & 4 & 0.11 & $1.00 \mathrm{E}-06$ \\
\hline GO:0007015 & actin filament organization & 111 & 12 & 2.4 & 2.30E-06 \\
\hline GO:0034446 & substrate adhesion-dependent cell spreading & 7 & 4 & 0.15 & 7.00E-06 \\
\hline GO:0006941 & striated muscle contraction & 8 & 4 & 0.17 & 1.40E-05 \\
\hline GO:0060361 & flight & 10 & 4 & 0.22 & 4.00E-05 \\
\hline GO:0007519 & skeletal muscle tissue development & 7 & 3 & 0.15 & 0.00033 \\
\hline \multicolumn{6}{|c|}{ Female abdomen downregulated } \\
\hline GO:0031122 & cytoplasmic microtubule organization & 20 & 5 & 0.31 & 9.80E-06 \\
\hline GO:0006270 & DNA replication initiation & 23 & 5 & 0.35 & 2.10E-05 \\
\hline GO:0007147 & female meiosis $\|$ & 5 & 3 & 0.08 & 3.40E-05 \\
\hline GO:0007338 & single fertilization & 27 & 6 & 0.41 & 0.00019 \\
\hline GO:0006277 & DNA amplification & 21 & 3 & 0.32 & 0.00023 \\
\hline GO:0007280 & pole cell migration & 21 & 4 & 0.32 & 0.00025 \\
\hline GO:0006013 & mannose metabolic process & 10 & 3 & 0.15 & 0.00038 \\
\hline GO:0071480 & cellular response to gamma radiation & 10 & 3 & 0.15 & 0.00038 \\
\hline GO:0006517 & protein deglycosylation & 13 & 3 & 0.2 & 0.00089 \\
\hline GO:0048640 & negative regulation of developmental growth & 67 & 5 & 1.02 & 0.00131 \\
\hline \multicolumn{6}{|c|}{ Female head upregulated } \\
\hline GO:0016059 & deactivation of rhodopsin mediated signaling & 16 & 3 & 0.04 & $6.60 \mathrm{E}-06$ \\
\hline GO:0042052 & rhabdomere development & 36 & 3 & 0.09 & $8.10 \mathrm{E}-05$ \\
\hline GO:0007601 & visual perception & 18 & 2 & 0.04 & 0.00083 \\
\hline GO:0045494 & photoreceptor cell maintenance & 29 & 2 & 0.07 & 0.00216 \\
\hline GO:0050830 & defense response to Gram-positive bacterium & 36 & 2 & 0.09 & 0.00331 \\
\hline GO:2000370 & positive regulation of clathrin-mediated endocytosis & 5 & 1 & 0.01 & 0.01202 \\
\hline GO:0051282 & regulation of sequestering of calcium ion & 5 & 1 & 0.01 & 0.01202 \\
\hline GO:0051966 & regulation of synaptic transmission, glutamatergic & 5 & 1 & 0.01 & 0.01202 \\
\hline GO:0050913 & sensory perception of bitter taste & 5 & 1 & 0.01 & 0.01202 \\
\hline GO:0007604 & phototransduction, UV & 5 & 1 & 0.01 & 0.01202 \\
\hline \multicolumn{6}{|c|}{ Female head downregulated } \\
\hline GO:0002181 & cytoplasmic translation & 86 & 28 & 1.78 & $9.10 \mathrm{E}-27$ \\
\hline GO:0055114 & oxidation-reduction process & 472 & 37 & 9.78 & 3.60E-10 \\
\hline GO:0046653 & tetrahydrofolate metabolic process & 5 & 4 & 0.1 & $8.70 \mathrm{E}-07$ \\
\hline GO:0000028 & ribosomal small subunit assembly & 12 & 5 & 0.25 & $2.50 \mathrm{E}-06$ \\
\hline GO:0006414 & translational elongation & 19 & 5 & 0.39 & $3.30 \mathrm{E}-05$ \\
\hline GO:0006730 & one-carbon metabolic process & 14 & 4 & 0.29 & 0.00015 \\
\hline GO:0006164 & purine nucleotide biosynthetic process & 83 & 5 & 1.72 & 0.00042 \\
\hline GO:0006635 & fatty acid beta-oxidation & 31 & 5 & 0.64 & 0.00134 \\
\hline GO:0017085 & response to insecticide & 12 & 3 & 0.25 & 0.00167 \\
\hline GO:0009620 & response to fungus & 54 & 4 & 1.12 & 0.00214 \\
\hline \multicolumn{6}{|c|}{ Male abdomen upregulated } \\
\hline GO:0045214 & sarcomere organization & 27 & 9 & 0.27 & 3.00E-12 \\
\hline GO:0030239 & myofibril assembly & 38 & 14 & 0.39 & $6.50 \mathrm{E}-09$ \\
\hline
\end{tabular}


Table 3 Ten most significantly enriched GO terms in each condition of the OR semispecies (Continued)

\begin{tabular}{|c|c|c|c|c|c|}
\hline GO Term & Annotation & $\begin{array}{l}\mathrm{Nr} \\
\text { Ann. }^{a}\end{array}$ & $\begin{array}{l}\mathrm{Nr} \\
\mathrm{DE}^{\mathrm{a}} \\
\end{array}$ & $\begin{array}{l}\mathrm{Nr} \\
\text { Exp. }^{a} \\
\end{array}$ & Signif. ${ }^{a}$ \\
\hline GO:0014866 & skeletal myofibril assembly & 7 & 4 & 0.07 & $3.40 \mathrm{E}-07$ \\
\hline GO:0006936 & muscle contraction & 23 & 8 & 0.23 & $9.80 \mathrm{E}-07$ \\
\hline GO:0060361 & flight & 10 & 4 & 0.1 & 2.00E-06 \\
\hline GO:0007629 & flight behavior & 25 & 5 & 0.25 & 4.30E-06 \\
\hline GO:0007498 & mesoderm development & 74 & 7 & 0.75 & $9.00 \mathrm{E}-06$ \\
\hline GO:0071688 & striated muscle myosin thick filament assembly & 5 & 3 & 0.05 & $1.00 \mathrm{E}-05$ \\
\hline GO:0007015 & actin filament organization & 111 & 8 & 1.13 & $1.60 \mathrm{E}-05$ \\
\hline GO:0006099 & tricarboxylic acid cycle & 34 & 5 & 0.35 & 2.10E-05 \\
\hline \multicolumn{6}{|c|}{ Male abdomen downregulated } \\
\hline GO:0032504 & multicellular organism reproduction & 943 & 35 & 23.13 & $6.50 \mathrm{E}-16$ \\
\hline GO:0055114 & oxidation-reduction process & 472 & 37 & 11.58 & $5.50 \mathrm{E}-11$ \\
\hline GO:0006508 & proteolysis & 677 & 33 & 16.61 & 1.50E-06 \\
\hline GO:0009631 & cold acclimation & 8 & 4 & 0.2 & $2.30 \mathrm{E}-05$ \\
\hline GO:0006629 & lipid metabolic process & 370 & 28 & 9.08 & $3.30 \mathrm{E}-05$ \\
\hline GO:0042364 & water-soluble vitamin biosynthetic process & 7 & 3 & 0.17 & 0.00047 \\
\hline GO:0042761 & very long-chain fatty acid biosynthetic process & 16 & 4 & 0.39 & 0.00051 \\
\hline GO:0045434 & negative regulation of female receptivity,postmating & 8 & 3 & 0.2 & 0.00074 \\
\hline GO:0006465 & signal peptide processing & 8 & 3 & 0.2 & 0.00074 \\
\hline GO:0005975 & carbohydrate metabolic process & 410 & 22 & 10.06 & 0.00187 \\
\hline \multicolumn{6}{|c|}{ Male head upregulated } \\
\hline GO:0055093 & response to hyperoxia & 7 & 1 & 0.01 & 0.0053 \\
\hline GO:0019731 & antibacterial humoral response & 28 & 1 & 0.02 & 0.0212 \\
\hline GO:0045793 & positive regulation of cell size & 29 & 1 & 0.02 & 0.0219 \\
\hline GO:0042052 & rhabdomere development & 36 & 1 & 0.03 & 0.0271 \\
\hline GO:0050830 & defense response to Gram-positive bacterium & 36 & 1 & 0.03 & 0.0271 \\
\hline GO:0030307 & positive regulation of cell growth & 40 & 1 & 0.03 & 0.0301 \\
\hline GO:0008286 & insulin receptor signaling pathway & 42 & 1 & 0.03 & 0.0316 \\
\hline GO:0018105 & peptidyl-serine phosphorylation & 42 & 1 & 0.03 & 0.0316 \\
\hline GO:0040018 & positive regulation of multicellular organism & 44 & 1 & 0.03 & 0.0331 \\
\hline GO:0046620 & regulation of organ growth & 49 & 1 & 0.04 & 0.0368 \\
\hline \multicolumn{6}{|c|}{ Male head downregulated } \\
\hline GO:0055114 & oxidation-reduction process & 472 & 45 & 10.14 & 4.40E-19 \\
\hline GO:1901606 & alpha-amino acid catabolic process & 35 & 7 & 0.75 & $\begin{array}{l}2.10 \mathrm{E}- \\
05\end{array}$ \\
\hline GO:0046653 & tetrahydrofolate metabolic process & 5 & 3 & 0.11 & $9.40 \mathrm{E}-05$ \\
\hline GO:0009620 & response to fungus & 54 & 5 & 1.16 & 0.00013 \\
\hline GO:0006730 & one-carbon metabolic process & 14 & 4 & 0.3 & 0.00017 \\
\hline GO:0019236 & response to pheromone & 15 & 4 & 0.32 & 0.00023 \\
\hline GO:0042559 & $\begin{array}{l}\text { pteridine-containing compound biosynthetic } \\
\text { process }\end{array}$ & 7 & 3 & 0.15 & 0.00032 \\
\hline GO:0072329 & monocarboxylic acid catabolic process & 40 & 3 & 0.86 & 0.00046 \\
\hline GO:0005977 & glycogen metabolic process & 15 & 5 & 0.32 & 0.00049 \\
\hline GO:0006098 & pentose-phosphate shunt & 8 & 3 & 0.17 & 0.0005 \\
\hline
\end{tabular}

${ }^{a} \mathrm{Nr}$ Ann. Number of times a GO term appears in the reference gene universe. $\mathrm{Nr} D E$ Number of DE genes which are annotated with the GO term. Nr Exp. Number of times a GO term would be expected to appear in the DE genes dataset. Signif Significance value in Fishers' test 
Table 4 Main biological functions associated with DE genes in the OR semispecies ${ }^{\text {a }}$

Gene Full gene name F

abd

\section{Metabolism - Lipids and fatty acids}

Acsl

ATPCL

CDase

Hmgs

Jhe

mag

Sc2

bgm

mino

SPLA2

Unannotated

wal

уір2

FarO

Metabolism - Purines

$\begin{array}{ll}\text { ade2 } & \text { adenosine } 2 \\ \text { Prat2 } & \text { Phosphoribosylamidotransferase } 2 \\ \text { Unannotated } & 2 \text { unannotated genes with homologs in D. melanogaster } \\ \text { Uro } & \text { Urate oxidase }\end{array}$

Metabolism - Amino acids

$\begin{array}{ll}\text { Hn } & \text { Henna } \\ \text { ppl } & \text { pumpless } \\ \text { Ssadh } & \text { Succinic semialdehyde dehydrogenase } \\ \text { Unannotated } & 6 \text { unannotated genes with homologs in D. melanogaster } \\ \text { Ahcy } & \text { Adenosylhomocysteinase } \\ \text { Sardh } & \text { Sarcosine dehydrogenase } \\ \text { Shmt } & \text { Serine hydroxymethyl transferase } \\ \text { Spat } & \text { Serine pyruvate aminotransferase } \\ \text { aay } & \text { astray } \\ \text { CG8129 } & \text { no_fullname } \\ \text { Nmdmc } & \text { NAD-dependent methylenetetrahydrofolate } \\ \text { P5cr-2 } & \text { dehydrogenase } \\ \text { mnd } & \text { Pyrroline-5-carboxylate reductase-like 2 } \\ \text { Gnmt } & \text { minidiscs } \\ \text { Gs2 } & \text { Glycine N-methyltransferase } \\ \end{array}$

\section{Metabolism - Carbohydrates and others}

Ilp8

LManIII

LManVI

AkhR

Galk

Ilp2

Taldo
Acyl-CoA synthetase long-chain

ATP citrate lyase

Ceramidase

HMG Coenzyme A synthase

Juvenile hormone esterase

magro

$\mathrm{sc2}$

bubblegum

minotaur

secretory Phospholipase A2

22 unannotated genes with homologs in D. melanogaster

$D / U$

D

yippee interacting protein 2

Fatty acyl-CoA reductase in oenocytes

U

D
D

D

D

D

D

D

D

D

D

D

D

D

D

U

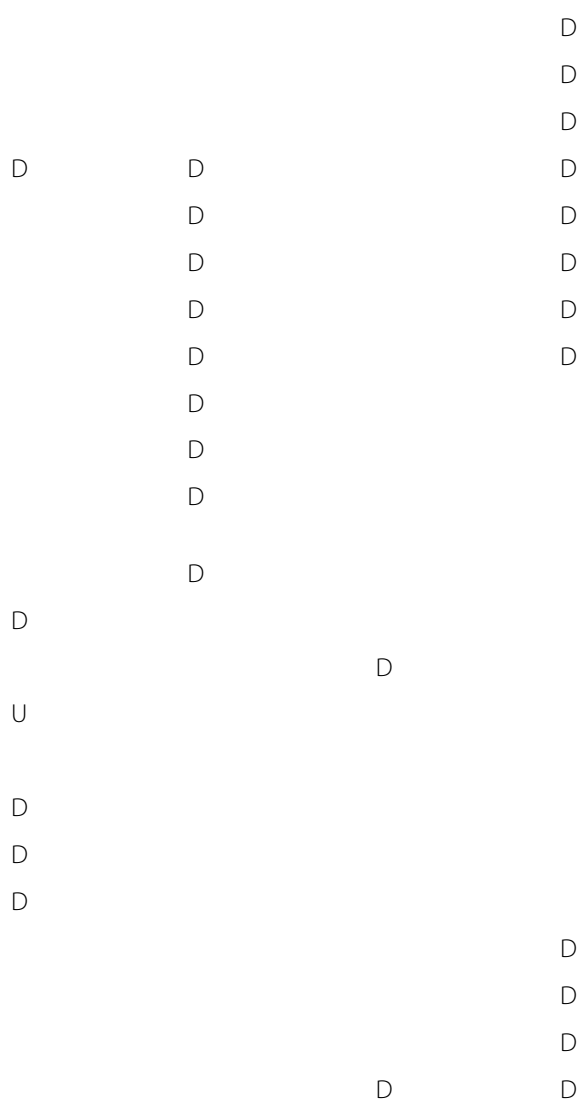


Table 4 Main biological functions associated with DE genes in the OR semispecies ${ }^{a}$ (Continued)

\begin{tabular}{|c|c|c|c|c|c|}
\hline Gene & Full gene name & $\begin{array}{l}F \\
a b d\end{array}$ & $\begin{array}{l}\mathrm{F} \\
\text { head }\end{array}$ & $\begin{array}{l}M \\
\mathrm{abd}\end{array}$ & $\begin{array}{l}M \\
\text { head }\end{array}$ \\
\hline AcCOAS & Acetyl Coenzyme A synthase & & $\mathrm{D}$ & $\mathrm{D}$ & $\mathrm{D}$ \\
\hline Hex-C & Hexokinase C & & $\mathrm{D}$ & $\mathrm{D}$ & $\mathrm{D}$ \\
\hline Idgf6 & Imaginal disc growth factor 6 & & $\mathrm{D}$ & $\mathrm{D}$ & $\mathrm{D}$ \\
\hline Mdh1 & Malate dehydrogenase 1 & & $\mathrm{D}$ & $\mathrm{D}$ & $\mathrm{D}$ \\
\hline Unannotated & 9 unannotated genes with homologs in D. melanogaster & & $\mathrm{D}$ & $\mathrm{D}$ & $\mathrm{D}$ \\
\hline LManll & Lysosomal alpha-mannosidase II & & $\mathrm{D}$ & & $\mathrm{D}$ \\
\hline pug & pugilist & & $\mathrm{D}$ & & $\mathrm{D}$ \\
\hline Pepck & Phosphoenolpyruvate carboxykinase & & $\mathrm{D}$ & & \\
\hline Amyrel & Amyrel & & & $\mathrm{D}$ & \\
\hline Cht4 & Chitinase 4 & & & $\mathrm{D}$ & \\
\hline Mal-A4 & Maltase A4 & & & $\mathrm{D}$ & \\
\hline $\ln R$ & Insulin-like receptor & & & U & \\
\hline LManl & Lysosomal alpha-mannosidase I & & & U & \\
\hline Cda5 & Chitin deacetylase-like 5 & U & & U & \\
\hline$k d n$ & knockdown & $U$ & & $U$ & \\
\hline rgn & regeneration & U & & U & \\
\hline Idgf4 & Imaginal disc growth factor 4 & U & & & \\
\hline boss & bride of sevenless & & U & & \\
\hline \multicolumn{6}{|l|}{ Proteolysis } \\
\hline LUBEL & Linear Ubiquitin E3 ligase & $\mathrm{D}$ & & $\mathrm{D}$ & \\
\hline $26-29-p$ & 26-29kD-proteinase & & & $\mathrm{D}$ & \\
\hline Ance & Angiotensin converting enzyme & & & $\mathrm{D}$ & \\
\hline cathD & cathD & & & $\mathrm{D}$ & \\
\hline iotaTry & iotaTrypsin & & & $\mathrm{D}$ & \\
\hline CtsB1 & Cathepsin B1 & & & $\mathrm{D}$ & $\mathrm{D}$ \\
\hline Acer & Angiotensin-converting enzyme-related & & $\mathrm{D}$ & & $\mathrm{D}$ \\
\hline Ance-5 & Ance-5 & & $\mathrm{D}$ & & $\mathrm{D}$ \\
\hline Unannotated & 30 unannotated genes with homologs in D. melanogaster & $\mathrm{D} / \mathrm{U}$ & $\mathrm{D}$ & $\mathrm{D}$ & $\mathrm{D}$ \\
\hline gol & goliath & & $\mathrm{D}$ & & \\
\hline Jon99Ci & Jonah 99Ci & $\mathrm{D}$ & D & & \\
\hline Ance-3 & Ance-3 & $\mathrm{D}$ & & & \\
\hline Bace & beta-site APP-cleaving enzyme & $\mathrm{D}$ & & & \\
\hline CG13025 & no_fullname & U & & & \\
\hline CG2224 & no_fullname & U & & & \\
\hline dmpd & dampened & U & & & \\
\hline$e(y) 2 b$ & enhancer of yellow $2 \mathrm{~b}$ & U & & & \\
\hline Jon65Aiv & Jonah 65Aiv & U & & & \\
\hline Jon66Cii & Jonah 66Cii & U & & & \\
\hline $\mathrm{Np} / 4$ & Nuclear protein localization 4 & U & & & \\
\hline SP1029 & SP1029 & U & & & \\
\hline Usp30 & Ubiquitin specific protease 30 & U & & & \\
\hline epsilonTry & epsilonTrypsin & U & & U & \\
\hline Prosalpha4 & Proteasome alpha4 subunit & & & U & \\
\hline
\end{tabular}


Table 4 Main biological functions associated with DE genes in the OR semispecies ${ }^{a}$ (Continued)

\begin{tabular}{|c|c|c|c|c|c|}
\hline Gene & Full gene name & $\begin{array}{l}F \\
a b d\end{array}$ & $\begin{array}{l}\text { F } \\
\text { head }\end{array}$ & $\begin{array}{l}\text { M } \\
a b d\end{array}$ & $\begin{array}{l}M \\
\text { head }\end{array}$ \\
\hline \multicolumn{6}{|l|}{ Immunity } \\
\hline Rel & Relish & & D & & \\
\hline Glt & Glutactin & & $\mathrm{D}$ & & $\mathrm{D}$ \\
\hline GNBP-like3 & GNBP-like 3 & & $\mathrm{D}$ & & $\mathrm{D}$ \\
\hline MP1 & Melanization Protease 1 & & D & & D \\
\hline GNBP2 & Gram-negative bacteria binding protein 2 & & & & $\mathrm{D}$ \\
\hline Tsf1 & Transferrin 1 & & & & $\mathrm{D}$ \\
\hline SPE & Spatzle-Processing Enzyme & & & D & $\mathrm{D}$ \\
\hline Spn28Dc & Serpin 28Dc & & & D & $\mathrm{D}$ \\
\hline yellow-f2 & yellow-f2 & & D & D & $\mathrm{D}$ \\
\hline casp & caspar & & & $\mathrm{D}$ & \\
\hline Hsp27 & Heat shock protein 27 & & & $\mathrm{D}$ & \\
\hline PPO2 & Prophenoloxidase 2 & & & $\mathrm{D}$ & \\
\hline CBP & sarcoplasmic calcium-binding protein & $\mathrm{D}$ & & & \\
\hline Dlip3 & Dorsal interacting protein 3 & $\mathrm{D}$ & & & \\
\hline Hat1 & Histone acetyltransferase 1 & $\mathrm{D}$ & & & \\
\hline heix & heixuedian & $\mathrm{D}$ & & & \\
\hline Unannotated & 4 unannotated genes with homologs in D. melanogaster & $U$ & $\mathrm{D}$ & U/ D & $\mathrm{D}$ \\
\hline AttA & Attacin-A & & & & $U$ \\
\hline LysD & Lysozyme D & & & U & \\
\hline Lmpt & Limpet & $U$ & & $U$ & \\
\hline AttD & Attacin-D & $U$ & $U$ & & \\
\hline DptB & Diptericin B & $U$ & $U$ & & \\
\hline AttC & Attacin-C & $U$ & & & \\
\hline e & ebony & $U$ & & & \\
\hline edin & elevated during infection & $U$ & & & \\
\hline Fer2LCH & Ferritin 2 light chain homologue & $U$ & & & \\
\hline Fuca & alpha-L-fucosidase & $U$ & & & \\
\hline pirk & poor Imd response upon knock-in & $U$ & & & \\
\hline Tep3 & Thioester-containing protein 3 & $U$ & & & \\
\hline Tep4 & Thioester-containing protein 4 & $U$ & & & \\
\hline yellow-b & yellow-b & $U$ & & & \\
\hline \multicolumn{6}{|c|}{ Perception - Olfactory } \\
\hline Est-6 & Esterase 6 & & D & & $\mathrm{D}$ \\
\hline Obp56a & Odorant-binding protein 56a & & $\mathrm{D}$ & & $\mathrm{D}$ \\
\hline Obp99c & Odorant-binding protein 99c & & $\mathrm{D}$ & & $\mathrm{D}$ \\
\hline Obp56g & Odorant-binding protein $56 \mathrm{~g}$ & & & & $\mathrm{D}$ \\
\hline Obp56h & Odorant-binding protein 56h & & & & $\mathrm{D}$ \\
\hline Obp83ef & Odorant-binding protein 83ef & & & & D \\
\hline Obp99b & Odorant-binding protein 99b & & & $\mathrm{D}$ & $\mathrm{D}$ \\
\hline Obp56d & Odorant-binding protein 56d & $U$ & & D & $\mathrm{D}$ \\
\hline \multicolumn{6}{|c|}{ Perception - Vision } \\
\hline ninaD & neither inactivation nor afterpotential D & & & & $\mathrm{D}$ \\
\hline chp & chaoptin & & U & & \\
\hline eys & eyes shut & & $U$ & & \\
\hline
\end{tabular}


Table 4 Main biological functions associated with DE genes in the OR semispecies ${ }^{a}$ (Continued)

\begin{tabular}{|c|c|c|c|c|c|}
\hline Gene & Full gene name & $\begin{array}{l}F \\
a b d\end{array}$ & $\begin{array}{l}\mathrm{F} \\
\text { head }\end{array}$ & $\begin{array}{l}\mathrm{M} \\
\mathrm{abd}\end{array}$ & $\begin{array}{l}\mathrm{M} \\
\text { head }\end{array}$ \\
\hline Fbxl4 & $\mathrm{F}$ box and leucine-rich-repeat gene 4 & & $u$ & & \\
\hline ninaC & neither inactivation nor afterpotential $C$ & & $u$ & & \\
\hline ninaG & neither inactivation nor afterpotential $\mathrm{G}$ & & U & & \\
\hline norpA & no receptor potential A & & $u$ & & \\
\hline prom & prominin & & $u$ & & \\
\hline
\end{tabular}

Pheromone production

$\begin{array}{ll}\text { Desat2 } & \text { Desaturase 2 } \\ \text { FASN1 } & \text { Fatty acid synthase 1 } \\ \text { FASN2 } & \text { Fatty acid synthase 2 } \\ \text { Fbp2 } & \text { Fat body protein 2 } \\ \text { Fbp1 } & \text { Fat body protein 1 }\end{array}$

$\begin{array}{ll} & D \\ & D \\ & D \\ D & D \\ D & D\end{array}$

\section{Reproduction - Females: Cell cycle, germline development}

\begin{tabular}{|c|c|}
\hline alphaTub67C & alpha-Tubulin at $67 \mathrm{C}$ \\
\hline aPKC & atypical protein kinase $\mathrm{C}$ \\
\hline $\mathrm{BicC}$ & Bicaudal C \\
\hline cad & caudal \\
\hline CDC45L & CDC45L \\
\hline cort & cortex \\
\hline CycB3 & Cyclin B3 \\
\hline dhd & deadhead \\
\hline egg & eggless \\
\hline exu & exuperantia \\
\hline $\mathrm{fs}_{\mathrm{s}}(1) \mathrm{Ya}$ & female sterile (1) Young arrest \\
\hline Fs(2)Ket & Female sterile (2) Ketel \\
\hline fzy & fizzy \\
\hline gcl & germ cell-less \\
\hline Grip84 & Gamma-tubulin ring protein 84 \\
\hline gus & gustavus \\
\hline hop & hopscotch \\
\hline lok & loki \\
\hline Mcm10 & Minichromosome maintenance 10 \\
\hline Mer & Merlin \\
\hline Mos & Mos oncogene \\
\hline nos & nanos \\
\hline $\mathrm{pbl}$ & pebble \\
\hline Pen & Pendulin \\
\hline pie & pineapple eye \\
\hline png & pan gu \\
\hline polo & polo \\
\hline slam & slow as molasses \\
\hline spd-2 & spindle defective 2 \\
\hline ssh & slingshot \\
\hline stai & stathmin \\
\hline swa & swallow \\
\hline tor & torso \\
\hline
\end{tabular}


Table 4 Main biological functions associated with DE genes in the OR semispecies ${ }^{\text {a }}$ (Continued)

\begin{tabular}{|c|c|c|c|c|c|}
\hline Gene & Full gene name & $\begin{array}{l}F \\
a b d\end{array}$ & $\begin{array}{l}\text { F } \\
\text { head }\end{array}$ & $\begin{array}{l}M \\
a b d\end{array}$ & $\begin{array}{l}M \\
\text { head }\end{array}$ \\
\hline Tre1 & Trapped in endoderm 1 & $\mathrm{D}$ & & & \\
\hline twe & twine & $\mathrm{D}$ & & & \\
\hline $\mathrm{Xpc}$ & Xeroderma pigmentosum $\% 2 \mathrm{C}$ complementation group C & $\mathrm{D}$ & & & \\
\hline asp & abnormal spindle & & $\mathrm{D}$ & & \\
\hline cmet & CENP-meta & & $\mathrm{D}$ & & \\
\hline spn-E & spindle E & & D & & \\
\hline Mdr49 & Multi drug resistance 49 & & & $\mathrm{D}$ & \\
\hline Rab1 & Rab1 & & & D & \\
\hline kug & kugelei & & & U & \\
\hline Doa & Darkener of apricot & $U$ & & U & \\
\hline bond & james bond & $U$ & & & \\
\hline$c(3) G$ & crossover suppressor on 3 of Gowen & $U$ & & & \\
\hline LanA & Laminin A & U & & & \\
\hline
\end{tabular}

Reproduction - Males: Regulation of postmating behavior

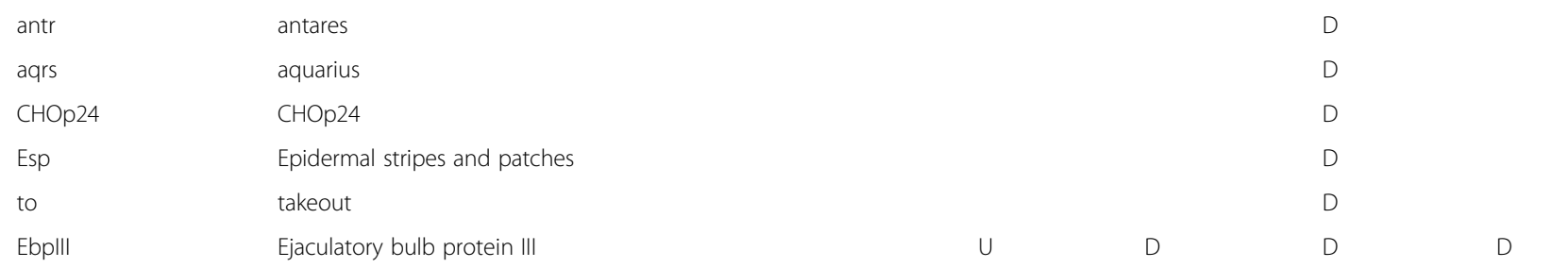

Muscular functions

$\begin{array}{ll}\text { Fkbp14 } & \text { FK506-binding protein 14 } \\ \text { Mical } & \text { Molecule interacting with CasL } \\ \text { SERCA } & \text { Sarco/endoplasmic reticulum Ca(2+)-ATPase } \\ \text { sesB } & \text { stress-sensitive B } \\ \text { skd } & \text { skuld } \\ \text { Actn } & \text { alpha actinin } \\ \text { bt } & \text { bent } \\ \text { clumsy } & \text { Clumsy } \\ \text { fln } & \text { flightin } \\ \text { Mhc } & \text { Myosin heavy chain } \\ \text { Mhcl } & \text { Myosin heavy chain-like } \\ \text { Mlc1 } & \text { Myosin alkali light chain 1 } \\ \text { Mlc2 } & \text { Myosin light chain 2 } \\ \text { Mlp60A } & \text { Muscle LIM protein at 60A } \\ \text { Msp300 } & \text { Muscle-specific protein 300 kDa } \\ \text { shot } & \text { short stop } \\ \text { Tm2 } & \text { Tropomyosin 2 } \\ \text { tn } & \text { thin } \\ \text { TpnC4 } & \text { Troponin C isoform 4 } \\ \text { uif } & \text { uninflatable } \\ \text { Unannotated } & \text { 28 unannotated genes with homologs in D. melanogaster } \\ \text { Unc-89 } & \text { Unc-89 } \\ \text { up } & \text { upheld } \\ \text { vkg } & \text { viking } \\ & \end{array}$

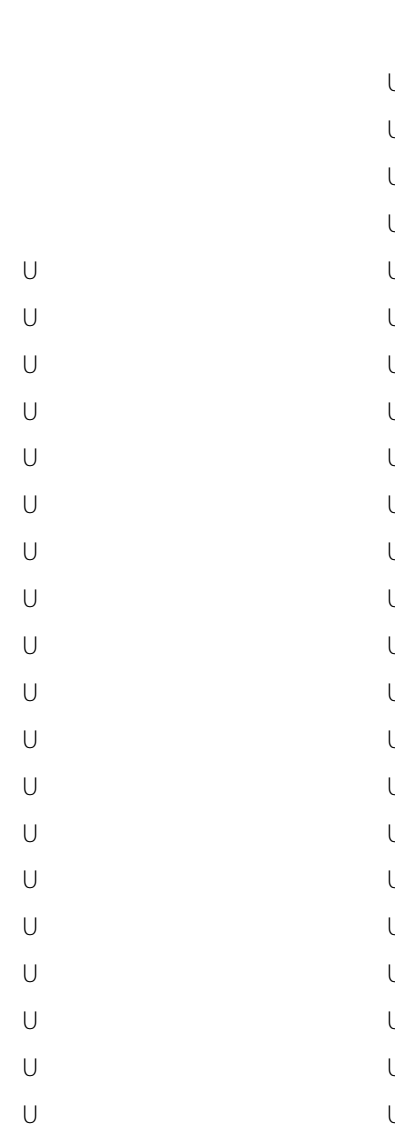


Table 4 Main biological functions associated with DE genes in the OR semispecies ${ }^{a}$ (Continued)

\begin{tabular}{|c|c|c|c|c|c|}
\hline Gene & Full gene name & $\begin{array}{l}F \\
a b d\end{array}$ & $\begin{array}{l}\text { F } \\
\text { head }\end{array}$ & $\begin{array}{l}\text { M } \\
\text { abd }\end{array}$ & $\begin{array}{l}M \\
\text { head }\end{array}$ \\
\hline Zasp52 & $\mathrm{Z}$ band alternatively spliced PDZ-motif protein 52 & $U$ & & $\mathrm{U}$ & \\
\hline Alk & Anaplastic lymphoma kinase & U & & & \\
\hline CAP & CAP & $U$ & & & \\
\hline chas & chascon & $U$ & & & \\
\hline Col4a1 & Collagen type IV alpha 1 & $U$ & & & \\
\hline eya & eyes absent & $U$ & & & \\
\hline Grip & Glutamate receptor binding protein & $U$ & & & \\
\hline if & inflated & U & & & \\
\hline Mlp84B & Muscle LIM protein at 84B & $U$ & & & \\
\hline Neurochondrin & Neurochondrin & $U$ & & & \\
\hline RyR & Ryanodine receptor & $u$ & & & \\
\hline sals & sarcomere length short & $U$ & & & \\
\hline TpnC73F & Troponin $\mathrm{C}$ at $73 \mathrm{~F}$ & $U$ & & & \\
\hline Zasp66 & Z band alternatively spliced PDZ-motif protein 66 & $U$ & & & \\
\hline \multicolumn{6}{|l|}{ Translation } \\
\hline Tfb4 & Transcription factor B4 & $\mathrm{D}$ & & & \\
\hline eEF1gamma & eukaryotic translation elongation factor 1 gamma & & $\mathrm{D}$ & & \\
\hline eEF2 & eukaryotic translation elongation factor 2 & & $\mathrm{D}$ & & \\
\hline eEF5 & eukaryotic translation elongation factor 5 & & D & & \\
\hline Rack1 & Receptor of activated protein kinase $\mathrm{C} 1$ & & D & & \\
\hline sta & stubarista & & $\mathrm{D}$ & & \\
\hline eEF1alpha1 & eukaryotic translation elongation factor 1 alpha 1 & & $\mathrm{D}$ & & $\mathrm{D}$ \\
\hline $\mathrm{RpL} / \mathrm{RpS}$ & 28 Ribosomal proteins & & $\mathrm{D}$ & D & $\mathrm{D}$ \\
\hline eEF1alpha2 & eukaryotic translation elongation factor 1 alpha 2 & $U$ & & U & \\
\hline \multicolumn{6}{|l|}{ Cytochrome P450 } \\
\hline Cyp311a1 & Cyp311a1 & & & $\mathrm{D}$ & \\
\hline Cyp4e2 & Cytochrome P450-4e2 & & & $\mathrm{D}$ & \\
\hline Сур6а8 & Cytochrome P450-6a8 & & & D & \\
\hline Cyp6d2 & Cyp6d2 & & & $\mathrm{D}$ & \\
\hline Cyp12a4 & Cyp12a4 & & D & & \\
\hline Cyp12d1-p & Cyp12d1-p & & $\mathrm{D}$ & & \\
\hline Cyp6t1 & Cyp6t1 & & $\mathrm{D}$ & & \\
\hline Cyp309a2 & Сyp309a2 & & $\mathrm{D}$ & & $\mathrm{D}$ \\
\hline Cyp6g1 & Cyp6g1 & & D & & D \\
\hline Cyp4p3 & Сyp4p3 & & $\mathrm{D}$ & D & $\mathrm{D}$ \\
\hline Cyp6d5 & Cyp6d5 & & $\mathrm{D}$ & $\mathrm{D}$ & $\mathrm{D}$ \\
\hline Cyp4ac2 & Cyp4ac2 & & & & $\mathrm{D}$ \\
\hline Cyp4e3 & Cytochrome P450-4e3 & & & U & \\
\hline \multicolumn{6}{|l|}{ Yolk proteins } \\
\hline Yp1 & Yolk protein 1 & & & & $\mathrm{D}$ \\
\hline Yp2 & Yolk protein 2 & & & & $\mathrm{D}$ \\
\hline Yp3 & Yolk protein 3 & & & & $\mathrm{D}$ \\
\hline
\end{tabular}

a $F$ Female, $M$ Male, Abd abdomen, $D$ Downregulated in WT, $U$ Upregulated in WT 
further indication of anabolic metabolism in GFR flies, as these conversions are mainly performed by enzymes involved in biosynthesis of amino acids and nucleotides.

We note that most of the above-mentioned expression changes manifest in heads and that glycine acts as a neurotransmitter which has both serine and proline as agonists. Several putative transporters of glycine and proline are encoded in Wolbachia genomes from various Drosophila species, and among the few genes for amino acid synthesis found in these genomes are those that can perform serine to glycine and threonine to glycine conversions.

\section{Metabolic hormones}

Both insulin-like peptide 2 and 8 have lower expression in WT flies, and the adipokinetic hormone (insect glucagon) receptor $(A k h R)$ has significantly lower expression in heads of male WT flies. Also the G-couple receptor encoded by boss has increased expression in heads of WT female flies. Boss is involved in regulation of sugar and lipid metabolism, and loss of function mutants show symptoms that resemble those of flies with defective insulin signaling [36]. Once again, this suggests that GFR flies have more nutrients available and a predominantly anabolic metabolism whereas WT flies have reduced nutrient availability and more catabolic metabolism.

\section{Proteolysis}

Although the GO term proteolysis is only enriched in downregulated genes in male abdomens, genes containing protease or peptidase domains are DE in various conditions, sometimes with high fold change. These DE genes are involved in various biological functions, but since the majority are downregulated in WT flies, the overall breakdown of proteins and consequent release of free amino acids appears to be lower in WT flies. One of the DE genes associated with proteolysis is a component of the proteasome (Proteasome alpha4 subunit). Proteins destined for degradation by the proteasome are tagged with ubiquitin, and a few genes involved in ubiquitination and deubiquitination, such as $L U B E L$, are also DE (Table 4).

Four different serpins (serine protease inhibitors) are downregulated in male abdomens, some of which are also DE in other conditions (Additional file 3). If these serpins inhibit proteases, this pattern indicates increased proteolysis in the presence of higher Wolbachia titer. However, two of them (Spn43Ab and Spn75F) are classified as non-inhibitory serpins and may have roles in reproduction, with $S p n 75 F$ being produced by the male accessory gland [37]. Of the remaining two, Spn28Dc inhibits spontaneous melanization and is necessary for pupal viability, while Spn42Da might be involved in retention of proteins in the endoplasmic reticulum [37].

\section{Immunity}

The effect of Wolbachia on immunity genes is a global response in the host, being observed in both sexes and tissues (Table 4).

One of the most important constituents of the insect immune system are the antimicrobial peptides (AMPs), small proteins which are active against a variety of bacteria, protozoans, fungi and viruses [38]. Several AMPs are upregulated with high fold changes in WT flies (Additional file 3), including three attacins and Diptericin B. Attacin A and Diptericin B are also upregulated in WT males of the AM semispecies (Additional file 5).

Wolbachia also affects the expression of several genes involved in the Toll and IMD pathways, which regulate AMP induction. DE components of the Toll pathway include two gram negative binding proteins (GNBP2 and GNBP-like3) and the Spatzle processing enzyme (SPE), all of which are downregulated in various conditions of WT flies (Table 4). GNBP-like3 is also downregulated in AM (female heads) and CA (female abdomens) flies, while the peptidoglycan recognition proteins $P G R P-S D$ and PGRP-SB1 are upregulated in AM WT flies (male heads and abdomens) (Additional files 4 and 5). Several regulators of the IMD pathway are also present among the DE genes and include Relish, which modulates expression of diptericins and attacins [39], heix, and the negative regulators caspar and SC2, all of which are downregulated in WT flies.

A number of DE genes are also associated with melanization, another important innate immune response in Drosophila. This is the case for Prophenoloxidase, MP1 and Yellow-f2, the latter is also DE in the CA semispecies (Table 4, Additional file 4). The immune gene edin, whose expression in the fat body is induced by bacterial infections [40], is upregulated with high fold change in OR WT flies and in males of WT AM flies (Table 4, Additional files 3, 4 and 5). Additionally, we identify DE genes linked to regulation of iron availability (Tsf1, Fer2LCH), response to fungus (Lmpt, CG9372) and opsonization (Tep3, Tep4) (Table 4).

\section{Perception}

Several genes associated with sensory perception are DE in multiple conditions, suggesting Wolbachia affects how $D$. paulistorum perceives its environment. This global response includes several odorant-binding proteins which are associated with the enriched GO terms "Response to pheromone", and "sensory perception of smell" (Table 3, Additional file 7). Most DE OBPs are downregulated in WT male heads (Table 4), a few of which with high fold change (Additional file 3). Additionally, Esterase 6, which affects the speed of odorant recognition [41], is downregulated in heads of WT flies of both sexes.

We also find several genes related to visual perception and eye development upregulated in female heads (Table 


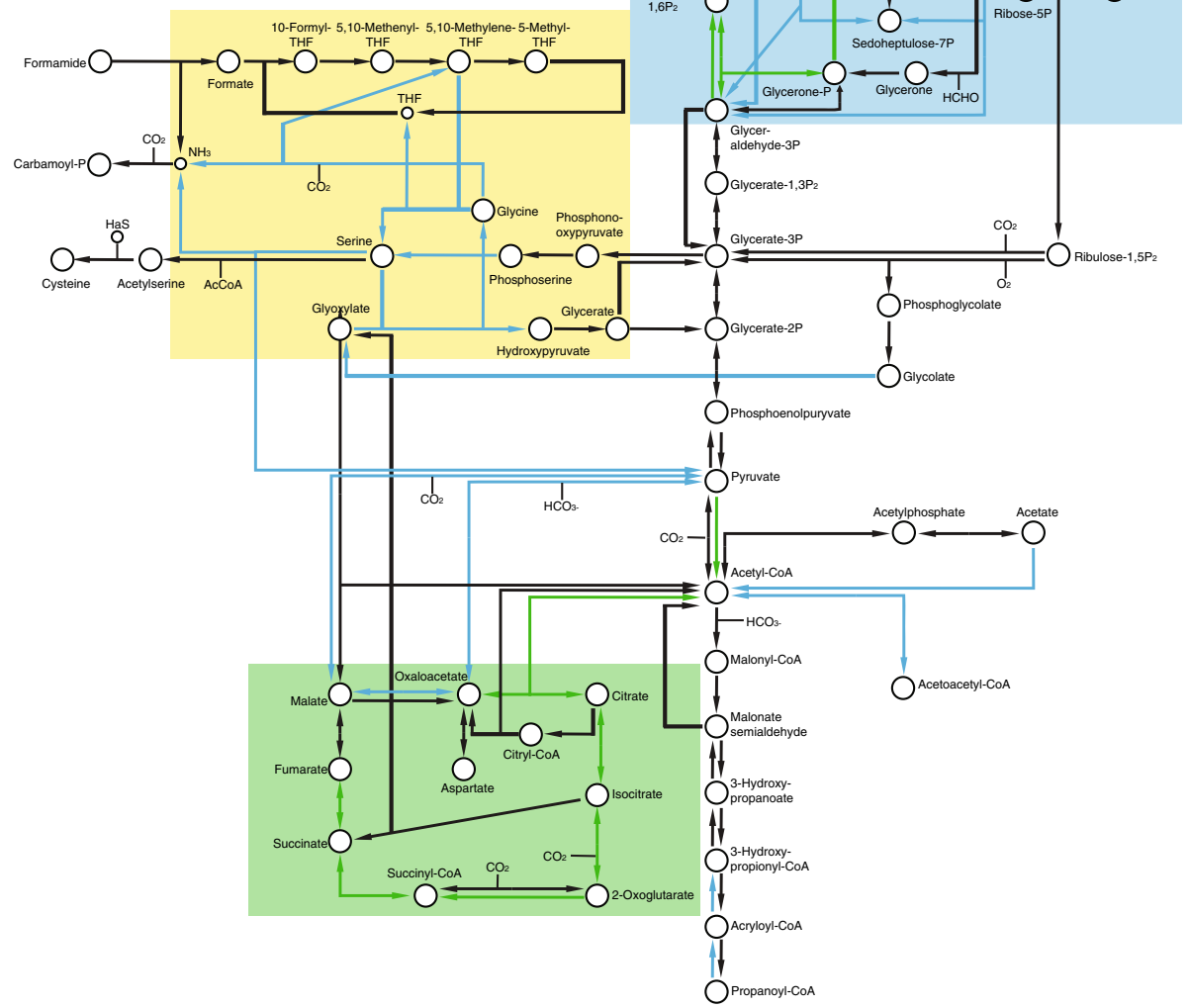

Fig. 4 Metabolic map of differentially expressed genes in carbon and energy metabolism of Drosophila paulistorum. Upregulated genes are shown in green and downregulated genes in blue. DE genes from all conditions of the OR semispecies are represented. Green box: TCA cycle. Yellow box: Glycine and serine metabolism. Blue box: pentose phosphate pathway. The figure is redrawn based on KEGG map01200

4), among which chaoptin, eyes shut and ninaG are DE with high fold changes (Additional file 3). Several GO terms associated with visual functions are enriched in the same condition (Table 3).

\section{Pheromone production}

Since cuticle hydrocarbon production is dependent on fatty acid metabolism, DE genes associated with such processes are candidates for affecting pheromone production. Among these, FASN1, FASN2 and desat2, all of which are downregulated in WT flies, have previously been implicated in Drosophila speciation (see Discussion). Other genes associated with fatty acid metabolism but so far with no described influence on pheromone synthesis are Bubblegum, which mediates activation of long chain fatty acids for synthesis and degradation of cellular lipids [42] and FarO, a fatty acyl-CoA reductase with activity in oenocytes, the cells which produce $\mathrm{CHCs}$ (Table 4). Also the fat body proteins Fbp1 and Fbp2, which participate in import of storage proteins into the fat body [43], might be involved in transport of pheromone precursors and are downregulated with high fold change in WT flies (Table 4, Additional file 3). Fbp1 is also DE in AM female abdomens (Additional file 5).

\section{Reproduction}

Many GO terms related to reproduction are enriched among downregulated genes in both male and female abdomens of WT flies (Table 3). In females, these involve several replication and cell-cycle-associated functions, as well as female-specific reproductive processes such as oogenesis, oocyte maturation, egg activation and germ cell migration. The DE genes in female abdomens 
associated with reproduction contribute to the high condition-specificity observed in this condition. In males, the enriched GO term with the largest number of reproduction-related genes is the broad "multicellular organism reproduction". Most genes in this category encode proteins of unknown functions that are inferred to be involved in reproduction through indirect evidence, for example by the fact that they are specifically expressed in the male accessory gland of $D$. melanogaster.

\section{Females}

Among the many reproduction-associated genes downregulated by Wolbachia in WT female abdomens are the two meiotic regulators Twine and Polo. Both are involved in activating Cyclin-dependent kinase 1 (CDK1)/ Cyclin B, which in turn is required for releasing the oocyte from the prophase I primary meiosis arrest $[44,45]$. CyclinB3, which has been shown to be involved in oocyte maturation, is downregulated. Furthermore, Cortex and Fizzy, two activators of the Anaphase promoting complex $(\mathrm{APC} / \mathrm{C})$ which is necessary for metaphase to anaphase transition, are downregulated in WT flies.

As a further indication of Wolbachia's effect on cell cycle, several genes involved in spindle formation and microtubule dynamics are also downregulated in WT flies. These include two subunits of the augmin complex that is involved in microtubule-dependent nucleation by recruitment of gamma-tubulin to the spindle (Table 4). Furthermore, Pan $g u$, implicated in the translational control of a large majority of mRNAs during egg activation, also has a lower expression in WT female abdomens, although the two activating proteins that usually form a complex with it are not affected.

Finally, since the first cell divisions after fertilization of the egg are controlled by maternal mRNAs and proteins, early embryonic development can also be affected by female DE genes. Several such genes are downregulated in WT female abdomens and include Deadhead, which is involved in male pronucleus activation after sperm entry into the egg, female sterile (1) and Young arrest, which is necessary for mitotic phase initiation during early embryogenesis. Additionally, many of the maternal effect genes involved in defining anterior-posterior polarity of the egg and embryo have lower expression in WT female abdomens. Nanos and caudal, two of the critical components for regulation of the posterior part of the embryo, as well as exuperantia and swallow, both of which interact with the anterior localization of bicoid mRNA, all have a lower expression in WT female abdomens (Table 4). Several other genes important for the development and migration of germ cells are also downregulated by Wolbachia, such as germ cell-less ( $g c l)$, whose low expression causes females to produce sterile offspring without germ cells [46], and gustavus (gus), involved in localizing vasa to the posterior end of the embryo and needed for primordial germ cell development [47].

\section{Males}

Several reproduction-related genes are DE in males, but only a handful have known functions. As in females, these genes are all downregulated in WT flies. Among the genes with known functions are several associated with post mating modulation of female receptivity and egg production. The two proteins aquarius and antares, for example, are necessary to facilitate the bond between sperm and sex peptide, a seminal protein known to increase production of eggs and decrease receptivity in mated females. Knocking down the expression of these genes in D. melanogaster males result in disturbed release of sperm from storage and reduced long term fertility in mated females [48].

The Angiotensin converting enzyme, Ance, suggested to have a role in spermatogenesis, has previously been considered a possible CI target [49]. Ance is downregulated in male WT abdomens while Ance-3 is upregulated in female WT abdomens, which supports the previous in vivo observation that Ance expression is higher in infected ovaries but lower in infected testes of $D$. simulans and D. melanogaster [49]. The genes Ance-5 and Acer (Angiotensin-converting enzyme-related), however, are here downregulated in the heads of WT flies of both sexes.

Two genes that affect male mating behavior are also downregulated in WT male abdomens. These are Takeout, a sex specific factor shown to influence courtship behavior in a non-pheromone dependent way [50] and Juvenile hormone esterase.

Finally, the Ejaculatory bulb protein III, a protein component of the posterior mating plug, is differentially expressed not only in male abdomens but in all sexes and tissues (Table 4).

\section{Muscular functions}

We find a large number of upregulated genes associated with enriched muscle-related GO terms in both male and female WT abdomens (Tables 3 and 4). Most of these encode structural components of the sarcomere, the basic unit of skeletal and cardiac striated muscles (Table 4), and many are DE with high fold change (Additional file 3). Other upregulated genes include the ryanodine receptor $(R y R)$, which appears $\mathrm{DE}$ in all three semispecies and is involved in calcium channeling, and the sarcoendoplasmic reticulum $\mathrm{Ca}^{2+}$ ATPase (SERCA), involved in muscle contraction (Table 4). The fact that Drosophila ventral abdominal muscles are innervated by glutamatergic synapses might also be the reason why genes associated with glutamate metabolism and 
reception such as Grip, Gs2 and clumsy, are upregulated in abdomens (Table 4). Taken together, these results indicate that Wolbachia is either directly or indirectly affecting muscle contraction.

\section{Translation}

A large number of translation-associated genes are downregulated in heads of both sexes, albeit in higher numbers in females where translation is also an enriched GO term (Table 3). In total, 36 ribosomal proteins and four elongation factors are downregulated by Wolbachia (Table 4) in WT flies, suggesting that Wolbachia reduces host translation at least in female heads.

\section{Cytochrome P450}

Cytochrome $\mathrm{P} 450$ is a family of heme-containing proteins which in D. melanogaster is associated with detoxification, production of the hormone 20-hydroxyecdysone and various behavioral and reproductive phenotypes [51]. In $D$. paulistorum, Wolbachia downregulates several cytochrome P450 genes, most of which are poorly characterized (Table 4). Studies with their orthologs in $D$. melanogaster suggest that Cyp311a1 is essential for larval development and that Cyp12d1-p, Cyp6g1, Cyp4s3, Cyp6a8 and Cyp12a4 have a role in detoxification, while defects in either Cyp4ac2 or Cyp4s3 lead to lower fitness. [51].

\section{Yolk proteins}

Three yolk proteins $(Y p 1, Y p 2, Y p 3)$ are downregulated in WT male heads, two of them with high fold change (Yp1, Yp3) (Additional file 3). The gene CG5966, downregulated with high fold change in WT female heads, has the same Lipase/Vitellogenin domains found in yolk proteins, which suggests it may be involved in similar responses in the female head.

\section{Does Wolbachia contribute to differences in semispecies- specific gene expression?}

In order to investigate if Wolbachia could contribute to speciation via changes in gene expression between semispecies, we mapped reads from all semispecies to the same transcriptome. The three assemblies show very high overlap with each other (Additional file 2: Figure S1), but since most of our analyses are focused on OR, we selected this transcriptome as reference. This choice is supported by the fact that the number of mismatches per base and the percentage of mapped reads obtained when mapping AM and CA to OR are similar to those seen when those semispecies are mapped to their own references (Additional file 1).

The PCA produced from these mappings showed that the three semispecies could be discriminated by their gene expression in both sexes and tissues (Fig. 5). The first and second principal components (PCs) separated the semispecies in all conditions except female abdomens, in which they were distinguished by $\mathrm{PC} 2$ and $\mathrm{PC} 3$ (Fig. 5, Additional file 2: Figure S6). We could not identify any particular factor associated with $\mathrm{PC} 1$ in the female abdomen.

The three semispecies were clearly separated in a PCA of the WT head samples, but were less clearly separated in the corresponding GFR plot (Fig. 5c, d, Additional file 2: Figures S7, S8). Additionally, we found that DE genes between WT and GFR head samples in the OR and AM semispecies are significantly overrepresented $\left(\mathrm{Chi}^{2}\right.$ test, $\mathrm{p}<0.01)$ among the genes that contribute the most to separating the semispecies in the PCA of WT head samples. Taken together, our results indicate that Wolbachia might contribute to the difference in expression pattern between heads of the three abdomens, although sex (PC1) explains $98 \%$ of the separation between the WT samples, a distinction between semispecies is seen when PC2 and PC3 are plotted (Additional file 2: Figure S9). Similar to what we detected in heads, DE genes between WT and GFR abdomen samples in the OR semispecies are overrepresented $\left(\mathrm{Chi}^{2}\right.$ test, $\left.\mathrm{p}<0.01\right)$ among the genes that contribute most to the semispecies separation. This suggests that Wolbachia may also contribute to gene expression differences between abdomens of the three semispecies, although in a more subtle way than in heads.

\section{Discussion}

Differential expression analysis between WT and GFR flies of the AM, CA and OR D. paulistorum semispecies revealed Wolbachia-induced changes on a wide range of host biological processes, particularly in the OR semispecies. Some of the most prominent effects are seen in metabolism, reproduction, immunity and muscular functions. Several differentially expressed genes possibly involved with production and reception of pheromones may have implications for host mating behavior and speciation.

The strategy of performing separate de novo transcriptome assemblies for AM, CA and OR was chosen in order to preserve potentially unique contigs of each semispecies. The relatively high percentage of ORFs which remained unannotated after similarity searches against both $D$. willistoni and D. melanogaster (Table 1) is likely an indication of sequence divergence between these species and $D$. paulistorum. This suggests de novo assembly as not only a suitable but potentially necessary approach. A high number of unannotated DE genes was found in both our analysis and in other systems [49, 52, 53], further showing the need for a de novo approach and also clearly demonstrating our incomplete understanding of the biology of Wolbachia-Drosophila interactions.

The use of different reference assemblies for the three semi-species also allowed us to detect a few DE genes 

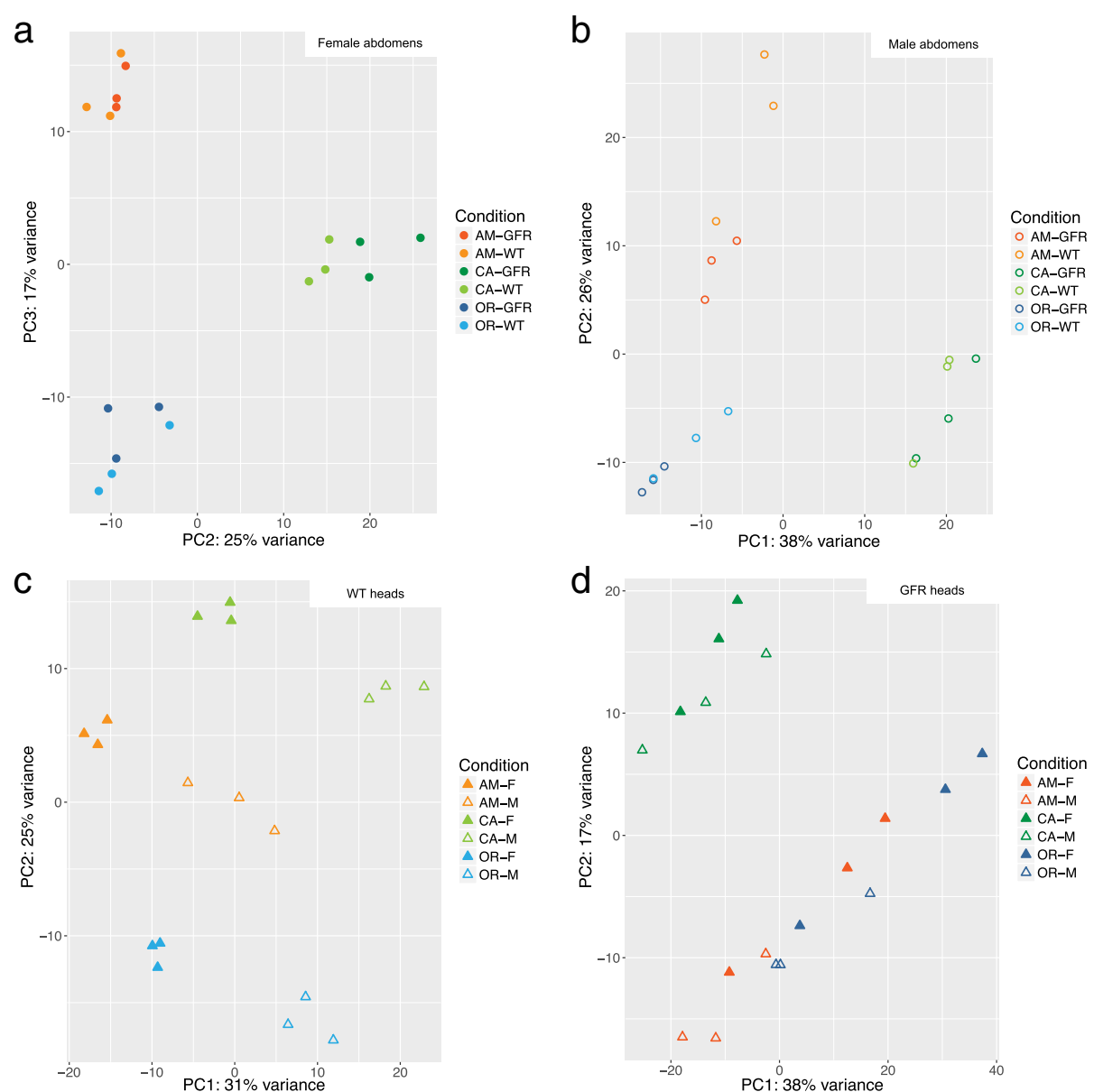

Fig. 5 Principal component analysis of all semispecies mapped to the OR transcriptome. (a): Female abdomens, (b): Male abdomens, (c): WT male and female heads, (d): GFR male and female heads. F: female, M: male, WT: wild type, GFR: gut flora restored, abd: abdomen

that are specific to a semispecies. The percentage of reads mapping back to the assembled transcriptomes, the proportion of complete ORFs and the number of BUSCO marker genes recovered indicate that the assemblies of all three semispecies are of high quality and completeness (Table 1).

\section{Influence of experimental setup and Wolbachia strain on differential expression results}

Although other studies have investigated the influence of Wolbachia on gene expression in insect hosts [49, 54, 55], none has analyzed the influence across different tissues and sexes separately. Using whole flies or cell lines might dilute the signal or blur the specificity of the biological response which, as we see from our results, is often tissue specific. Thus, using multiple tissues and both sexes is expected to be a more precise strategy for transcriptome studies between Wolbachia and host. As a likely result of this method, the $1192 \mathrm{DE}$ genes found in OR (Table 2) are considerably more than the 250-450
DE genes reported in a number of previous studies [49, 52-54].

On the other hand, the numbers of DE genes for AM and CA (Table 2) are in line with other studies of Wolbachia and host gene expression, and consequently lower than those in OR. A likely explanation for the smaller number of DE genes in AM and CA is the low Wolbachia infection titer in these two semispecies compared to OR [8]. This difference in Wolbachia titer is illustrated by the number of reads and ORFs associated with Wolbachia in each semispecies (Table 1, Additional file 1 ) but is also known from previous studies $[8,23]$. In the lower titer infections, it is possible that extracting RNA from whole heads and abdomens might have diluted the signal, since probably only a small number of cells are infected with Wolbachia. Hence, analyzing infected and uninfected cells separately might thus be necessary in order to detect differential expression in low titer infections. Alternatively, it is also possible that the observed differences between AM, CA and OR are a consequence of the three semispecies being infected 
with different Wolbachia strains. Previous studies have shown that which host genes are differentially expressed in response to Wolbachia can differ according to the infecting Wolbachia strain [52]. Unfortunately, we currently do not know what the genetic differences between the strains are, and are therefore unable to test or speculate further about this.

Given that we don't fully understand the biological reason behind the observed differences between the semispecies, we can't completely discard the hypothesis that the lower numbers of DE genes in AM and CA also mean a reduced or divergent impact of the symbiont on these hosts compared to OR. However, we notice that several functional categories are enriched in all the semispecies (Table 3, Additional file 7). This fact not only supports previous observations that low titer AM and CA Wolbachia have a relevant impact on host biology $[8,11,12,23]$, but also suggest similarities on host effects in the three semispecies. In AM, this is seen as upregulated muscle functions in male abdomens, upregulated visual function in the female heads, upregulated defense genes in male and female heads and downregulated metabolic processes in female heads (Additional file 7). In CA, we observe upregulation of immunity genes in male abdomens and downregulation of carbohydrate metabolism in female abdomens (Additional file 7). Interestingly, even though these functional categories are affected in multiple semispecies, only a small overlap exits in the actual DE genes (Fig. 2). This again suggests a relatively high specificity in the interactions between each Wolbachia and its D. paulistorum host.

In the next sessions, we discuss the main biological functions affected by Wolbachia in the D. paulistorum host. Both previously known and novel/putative functions are discussed and, whenever relevant, we consider how the affected genes might support the hypothesis that Wolbachia contributes to host speciation.

\section{Functions previously known to be affected by Wolbachia Metabolism}

Given its obligate intracellular lifestyle and small genome with limited gene content, it is clear that Wolbachia is not able to produce all the nutrients it needs and thus must obtain them from the host. As a likely consequence of this, we find that many genes involved in metabolic and biosynthetic processes are DE between WT and GFR flies. High Wolbachia levels are associated with upregulation of genes in the TCA cycle and a generally more catabolic metabolism. Low Wolbachia levels, on the other hand, have increased expression of genes involved in beta-oxidation and the pentose phosphate pathway, an indication of anabolic metabolism and active production of precursors for nucleotide, amino acid and lipid biosynthesis. These differences in gene expression are similar to those observed in protein expression of D. melanogaster on a poor vs. a rich diet [56], suggesting that being infected with Wolbachia may have a significant metabolic cost for the host.

In contrast to what we described for genes in glucose and energy metabolism, several of the downregulated genes associated with amino acid metabolism were recently shown to have a higher expression in starved compared to non-starved brains of D. melanogaster [57]. The upregulation of these genes is correlated with high serine levels and starvation-induced sleep suppression [57]. Hence, the expression pattern of the serine and glycine metabolism genes in heads of GFR flies, rather than WT flies, mimic starvation conditions. Interestingly, in line with this observation, a recent study showed an increase in nighttime activities in non-Wolbachia infected D. melanogaster flies compared to infected [58].

Several studies have shown that Wolbachia rely on cholesterol from the host, a property which is also believed to be important for the pathogen blocking phenotype of the symbiont $[59,60]$. We observe that many genes involved in fatty acid and lipid metabolism are downregulated in WT flies, including some that encode proteins with different abundances in Wolbachia-infected and uninfected mosquito cells [60]. Additionally, several putative cholesterol ester hydrolases responsible for making cholesterol and free fatty acids available to the cell when they are required for membrane and lipoprotein formation are downregulated in WT flies. Recent work on the human pathogenic bacterium Chlamydia trachomatis has demonstrated that cholesterol esterification is likely essential for cholesterol import into the membrane inclusion where the bacterium resides [61]. It is thus possible that the downregulation of genes that hydrolyze cholesterol esters in WT flies reflects a need for these molecules also by Wolbachia.

\section{Reproduction}

Wolbachia downregulates several genes involved in cell cycle, oocyte development, germ cell development and germ cell migration in OR female abdomens. Differential expression of these could potentially lead to phenotypes that are lethal for the embryo or which may cause defects in ovary development. An example of this is Deadhead, which is necessary for proper paternal chromatin decondensation during fertilization [62]. Interestingly, mutant maternal Deadhead can result in haploid embryonic development due to failed paternal chromatin condensation, a condition that resembles the CI phenotype induced by Wolbachia [32]. Further studies are still necessary to investigate whether $\mathrm{CI}$ occurs in $D$. paulistorum, but differential expression of such genes suggest 
Wolbachia might influence postmating compatibility in this species either through CI or other mechanisms.

\section{Immunity}

Our results show that Wolbachia influences the expression of genes associated with a wide range of immune responses in $D$. paulistorum. Among these, a clear pattern is seen on AMPs, which are consistently upregulated in WT flies, often with high fold change. Genes associated with melanization, opsonization, regulation of Toll and IMD pathways and control of nutrient availability are also affected, although with variable intensity and direction of regulation.

Different studies have linked symbionts in general and Wolbachia in particular to effects on the insect immune system. Tsetse flies, for example, become heavily immunocompromised if cleansed of their primary symbiont Wigglesworthia [63, 64], and mosquitoes develop increased resistance to viruses, bacteria, nematodes and protozoans when transinfected with Wolbachia [65]. Likewise, natural Wolbachia infections are known to provide protection against viruses and bacteria in Drosophila [66-68], although the mechanisms involved are not fully understood. Current hypotheses suggest the symbiont may directly or indirectly promote immune priming [65], activate the Toll and IMD pathways [69], or induce production of detoxifying agents and AMPs [70].

The upregulation of AMPs in WT D. paulistorum (Table 4, Additional files 3, 4 and 5) corroborates similar observations previously made in D. melanogaster and mosquito cell lines $[49,52,53,55]$. Although this increase in AMP expression may be an infection-mediated immune response, it is also possible that the host needs to produce more of these molecules to control the number and localization of Wolbachia cells. In D. paulistorum, Wolbachia is localized in highly defined tissues and cell types such as the embryonic primordial germ cells [8], specific brain regions [17] and oenocytes [23]. Thus, one can hypothesize that AMPs could be used by the host to create and maintain this pattern in a similar way to what is observed in the weevil Sitophilus zeamais, which uses AMPs to restrict its bacterial endosymbiont to bacteriocytes. [71]. The fact that AMPs interact directly with their targets in a concentration dependent way could also explain why these molecules are generally DE with higher fold change than other immune genes, as larger changes in expression would be necessary for creating biologically relevant variations in their effect. Elevated levels of the AMP Diptericin $B$ and of the immune gene GNBP-like3 have also been recently correlated with enhanced long-term memory in D. melanogaster [72] suggesting that Wolbachia-mediated higher expression of AMPs in D. paulistorum might, directly or indirectly, improve host memory and thereby possibly affect sexual behavior [8]. It's also worth noting the differential expression of genes associated with the Toll and IMD pathways, which mediate AMP production. Although only a few of the constituents of the pathway are affected, they might still have a relevant role in host immunity given their regulatory functions.

Finally, we also find several DE genes in OR associated with melanization, suggesting that the increase in this response induced by $w$ MelPop in female mosquitos [73] could also be induced in $D$. paulistorum by its native Wolbachia infection.

\section{Translation}

The downregulation of ribosomal proteins and elongation factors in heads of OR WT flies suggests that Wolbachia suppresses host translation. Similar effects have been observed on the protein level in Wolbachia-infected D. melanogaster and D. simulans ovaries [74] and it is possible that they arise as a consequence of symbiont-mediated metabolic changes. Translation initiation is inhibited when the cell lacks essential amino acids such as leucine and methionine [75], hence, if severe enough, the appropriation of amino acids by $\mathrm{Wol}$ bachia could possibly reduce overall translation. However, it is unclear if such a lack of amino acids could result in a reduced expression of the ribosomal proteins and other genes involved in translation as observed here. Recent work suggests that Wolbachia titer increases if host translation is blocked [55], thus one possibility is that Wolbachia lowers translation in D. paulistorum heads in order to attain the high infection titer observed in the brain of this species [17].

\section{Novel functions affected by Wolbachia Muscular functions}

An unexpected number of genes related to muscular functions are upregulated by Wolbachia in both male and female abdomens. Wolbachia is known to infect muscles in Drosophila [18] and to increase locomotion in mosquitoes [76], but those observations are related to thoracic and not abdominal muscles, which is what we analyze here. One possibility is that Wolbachia might affect the heart, which in Drosophila is one of the largest skeletal muscles in the abdomen. Reduced expression of sarcomere genes has been connected to various cardiac diseases in Drosophila [77], and removing Wolbachia and thereby lowering the expression of such genes could possibly cause disease and lowered fitness in the flies. However, currently no phenotype connects Wolbachia and heart disease.

Although relatively little is known about the functions of abdominal muscles in Drosophila [78], one might speculate that altered muscle function could have 
implications in movement patterns associated with courtship and reproduction. The ventral abdominal muscles (VAMs), for example, are necessary for proper folding movements of the abdomen [78], and male Drosophila are known to use abdominal vibrations during courtship [79]. Hence, it is possible that changes in VAM activities could affect mating success. Another possibility is that the muscular genes observed to be $\mathrm{DE}$ in the abdominal muscles are also DE in thoracic muscles. If so, wing muscle function might be affected and have an impact on the generation of "love songs" by male flies. These songs are produced by rapid wing vibrations and have crucial role in Drosophila courtship by affecting female receptivity [79].

Finally, the large number of DE genes with musclerelated functions could also be a result of Wolbachia's effect on host metabolism, since starvation induces a set of behavioral changes in Drosophila that enhances the search for food [80]. This behavioral change occurs through modulated perception of odors and tastes [81] as well as increased locomotor activity, which leads to a higher chance of finding food. One possibility is thus that the increased expression of muscle related genes might indicate that locomotion is increased in flies with WT levels of Wolbachia, possibly as a result of malnutrition. Again, we would have to assume that thoracic muscles are also affected, as much of the locomotion is supported by these muscle groups. Contrary to this hypothesis, though, the adipokinetic hormone (insect glucagon) receptor $(A k h R)$ required for starvation-induced activity [80] has a significantly lower expression in WT flies, whereas the insulin-like receptor that was seen to counteract $A k h R$-induced locomotion, is instead upregulated. This is the opposite pattern of what would be expected if WT titers of Wolbachia lead to starvationinduced behavior.

Overall, an effect of Wolbachia on muscles, either directly or as a byproduct of metabolic changes, might impact courtship behavior and thus conceivably lead to the emergence of assortative mating.

\section{Pheromone production and reception}

Most DE genes involved in pheromone production participate in $\mathrm{CHC}$ synthesis and have a role in fatty acid metabolism. Among these, the fatty acid synthase FASN2 has been implicated in the reproductive isolation between D. serrata and D. birchii [82]. In that case, selective pressure for different cuticle composition in populations living in contrasting humidity conditions probably led to divergence, as $\mathrm{CHCs}$ have a dual role as cuticle constituents and pheromones [82]. In an analogous way, one can hypothesize that Wolbachia-induced changes to fatty acid metabolism could affect the expression of FASN in D. paulistorum, leading to premating isolation between populations that respond differently to Wolbachia or which carry distinct symbiont strains. Importantly, FASN2 is one of the few genes that are DE in all three semispecies (Fig. 2, Additional file 6). The influence of Wolbachia on D. paulistorum chemical communication is exemplified by a recent study showing that reduction of Wolbachia titer in males significantly affect semispecies-specific $\mathrm{CHC}$ profiles and triggers assortative mating of WT females against the symbiontdepleted mates [23].

Other fatty acid-related genes known to affect pheromone production in Drosophila are desat1 and desat2, the latter of which is downregulated in CA and OR male WT abdomens. Desaturases create double bonds in $\mathrm{CHC}$ molecules, thus influencing the proportion of different compounds in the fly pheromone mix $[26,83]$. desat1 has been shown to affect sex pheromones of $D$. melanogaster [84], and both desat1 and 2 are likely implicated in incipient speciation in the same species [83].

Mechanisms for pheromone reception in Drosophila are generally poorly understood, but at least one OBP, $L U S H$, has been linked to responses to the courtship pheromone 11-cis-vaccenylacetate in $D$. melanogaster $[85,86]$. Thus, the differential regulation of seven OBPs in OR flies can possibly affect pheromone response and consequently mate choice. Another protein involved in pheromone perception is the odorant degrading protein Esterase 6, here downregulated in heads of WT OR flies. It degrades odorants after they have bound to a receptor, thus allowing faster interaction with new molecules [41, 87].

Overall, the majority of the DE genes likely to be associated with pheromone production and reception are downregulated in OR WT heads and male abdomens, but a small number is upregulated in WT female abdomens. It is not clear why the direction of regulation in female abdomens is opposite to heads and male abdomens, but this pattern is seen in fatty acid metabolism genes, OBPs and Esterase 6, suggesting a biological reason might exist.

\section{Vision}

We found that several genes related to visual functions are upregulated in female heads of WT OR flies. Wolbachia is known to infect the optic lobe and the retina of D. melanogaster [18], and recent work in D. paulistorum showed that it also infects areas of the brain responsible for sensorial responses in that species, including vision [17]. It is not clear what biological consequences this has for the host, but one possibility is that it affects reproductive behavior, as vision has a documented importance in recognition of potential mates and perception of locomotor cues during Drosophila courtship [88, 89]. 


\section{Yolk proteins}

The Wolbachia-induced upregulation of yolk proteins in heads of WT D. paulistorum is rather intriguing given the usual association of these proteins with vitellogenesis. In female D. melanogaster, Yp1-3 produce most of the components of egg yolk and are positively correlated with fertility, while in males they are implicated in sperm processing [90, 91]. Functions in the head are not known, although an association with the head fat body has been observed in D. melanogaster [92]. In the same species, yolk proteins are known to interact with the insect hormone ecdysone and to negatively impact longevity of both sexes [91]. So far, there is no clear connection between Yp1-3 and any known Wolbachia phenotype, but it is interesting to note that the three yolk proteins are among the genes contributing the most to the separation between semispecies in the PCA of WT heads (Fig. 5).

Does Wolbachia play a role in D. paulistorum speciation? Mating between WT flies of different $D$. paulistorum semispecies has been shown to result in very low reproductive success, hybrid male sterility, and high rates of embryonic lethality $[4,8,9,11,12]$. In such conditions, it is expected that mechanisms would arise allowing individuals to recognize compatible mates before they waste energy and resources on unsuccessful reproductive attempts [93, 94]. Wolbachia most likely also benefits from preventing unfruitful host matings, as these are dead ends for a vertically transmitted symbiont. It seems plausible, then, that both host and symbiont would benefit if Wolbachia could enhance discrimination between $D$. paulistorum semispecies by inducing or enhancing some form of premating incompatibility.

Although our data doesn't allow us to make definite conclusions regarding a role of Wolbachia in D. paulistorum speciation, especially in the case of low titer infections, several of our results support that hypothesis. Host premating isolation, for example, could be affected by DE genes involved in pheromone production and reception, as those might interfere with chemical communication. Genes associated to muscular functions might influence mating locomotor activities, including production of "love songs" through wing vibrations and abdominal tapping. Finally, genes which affect vision could impact recognition of mating cues and partner identification. The fact that genes affected by Wolbachia are overrepresented among those that contribute most towards distinguishing the gene expression between heads of AM, CA and OR flies (Fig. 5) also suggests that the symbiont might contribute to the emergence of behavioral differences between the semispecies, possibly including mate recognition.
Postmating isolation, on the other hand, could be influenced by many of the reproduction genes associated with cell cycle and germ cell development, as a disruption of their usual expression pattern could potentially harm or prevent embryonic development.

The strong metabolic changes observed in D. paulistorum as a result of Wolbachia infection also lead to the hypothesis that some or all of the functions with putative effects on speciation are a consequence of altered host metabolism. If correct, the metabolic cost of carrying Wolbachia might have causes physiological alterations which in turn impact reproductive behavior. Ultimately, those changes might have led to pre- and postmating isolation and speciation.

\section{Conclusions}

The obligate relationship between $D$. paulistorum and Wolbachia combined with the ongoing divergence in the D. paulistorum complex results in a unique system for investigating symbiont-mediated speciation. Our results show that Wolbachia affects gene expression in different ways in two tissues and both sexes of three semispecies of $D$. paulistorum. The effect is particularly strong in OR, potentially due to the higher infection titer compared to the AM and CA semispecies.

Genes affected by Wolbachia are linked to a wide variety of biological functions. Some are globally responsive and previously known to be affected by the symbiont, such as immunity, reproduction and metabolism, while a few are novel tissue- or condition-specific functions, like those associated with muscles and vision. Our findings suggest that the competition between host and symbiont for amino acids, carbohydrates and lipids can be the cause of several of the physiological changes observed in D. paulistorum and that the association with Wolbachia either requires or leads to adjustments in the host immune functions.

We show that Wolbachia contributes to making the gene expression in heads more distinct between semispecies, supporting the hypothesis that the symbiont might influence mate choice and modulate host behavior. Furthermore, we suggest that a role for Wolbachia in the speciation of $D$. paulistorum is supported by the differential expression of genes involved in pheromone production and reception as well as reproduction and early embryonic development, as these are likely to influence pre- and postmating isolation between semispecies. It remains to be tested whether Wolbachia is a driving force of the speciation process or if it reinforces an already ongoing trend. In either case, we hypothesize that the possible contribution of Wolbachia to D. paulistorum semispecies isolation could be a benefit that maintains the infection in spite of the metabolic costs, 
as it might ultimately increase the chance of a fly identifying a suitable mate.

\section{Materials and methods}

Flies were kept in Wolfgang Miller's lab at the Medical University of Vienna and belong to three of the classical semispecies of D. paulistorum: Orinocan - line O11, Amazonian - line A28 and Centro American - line C2. These lines were obtained from Lee Ehrman, and descend from flies used in the experiments which helped define the classical $D$. paulistorum semispecies, back in the 1960s [5]. Flies were reared on Formula 4-24 ${ }^{\circ}$ instant food at $21-22^{\circ} \mathrm{C}$ and 12 hour light/ dark cycle.

\section{Antibiotics treatment and gut flora restoration}

In order to knock down the Wolbachia infection, WT flies were kept on food containing Rifampicin $0.2 \%$ w $/ \mathrm{v}$ for three consecutive generations according to [8]. PCR screenings targeting the Wolbachia wsp gene showed that infection titer was reduced to below detection level after treatment. Gut flora restoration was done by transferring treated flies to tubes containing regular food in which virgin WT females of the corresponding semispecies had been kept for 2-3 days, so that feces had accumulated on the food and inner surfaces. After egg deposition, adults were removed and the larvae which developed in those vials were considered gut flora restored.

\section{Sample collection and RNA extraction}

Whole heads, including brain and mouthparts, and abdomens, containing both gonads and gut, were severed from 3-day old adult females and males of either WT or F3 generation GFR flies using fine tweezers. No attempt was made to keep flies virgins. A total of approximately 20 heads and 10 abdomens were pooled per head and abdomen sample, respectively. Three biological samples were collected per condition and RNA was subsequently extracted using a TRIzol ${ }^{\mathrm{Tm}}$ Reagent protocol (Sigma). In brief, samples were homogenized in $\mathrm{TRIzol}^{\mathrm{T \omega}}$ reagent with sterile pestles, incubated at room temperature, and phases were separated using 1-bromo-3-chloropropane (BCP) phase separation reagent. RNA was collected from the aqueous phase, transferred into new tubes and precipitated with isopropanol. Pellets were washed, dried and rehydrated in RNase free water.

\section{Sequencing and read quality control}

The extracted RNA was used for library preparation at the SNP\&SEQ Technology Platform in Uppsala, Sweden. Per sample, a total of $0.5 \mu \mathrm{g}$ of RNA was treated with the ScriptSeq complete Gold Epidemiology kit (Illumina, part\# BEP1224) for rRNA depletion according to the manufacturer's protocol (Lit\#356-4-2013 RevA). The kit was originally designed for human, mouse, rat and bacterial samples, but successful use in Drosophila is reported at the manufacturer's species compatibility table. Sequencing was done with Illumina HiSeq2500, to produce paired-end 125 bp reads using v4 sequencing chemistry. All 72 samples were run in the same Illumina flowcell. Samples from the different conditions (sex, treatment, semispecies) were arranged so that replicates of the same condition were run in different lanes and with molecular identifiers rotated between samples to avoid any systematic bias.

Sequenced reads were quality-checked with FastQC v0.10.0 [95] and processed with Trimmomatic v0.36 [96] for residual adapter removal and mild quality trimming using the parameters: ILLUMINACLIP:TruSeq3-PE.fa:2:40:15 LEADING:3 TRAILING:3 SLIDINGWINDOW:4:15 MINLEN:95

\section{Transcriptome assembly and ORF prediction}

Transcriptomes of each semispecies were separately assembled with Trinity v2.1.1 [97] using the parameters "--min_kmer_cov 2" and "--normalize_reads". All samples of each semispecies were used for the respective assembly with the exception of O11-GFR-F-abd3 and O11-WT-M-abd3, for the OR assembly, and C2-WTabd1, for CA assembly, which contained higher than average rRNA level as indicated by the reports from the sequencing facility. Contigs within one Trinity gene group are referred to as "genes" as they likely represent isoforms or possible assembly artefacts from the same gene.

Transcriptome completeness was evaluated with BUSCO v3.0.2 [98] using Arthropoda, Insecta and Diptera markers. Assembly quality was further assessed through the percentage of reads mapping back to their respective assembly using BWA mem aligner [99] and by calculating N50, average contig length and percentage of complete ORFs.

An estimate of the percentage of reads mapping to different organisms was obtained for each assembly through competitive mapping with BWA mem to a reference including genomes of Drosophila, Wolbachia, yeast and Drosophila gut bacteria (Additional file 1).

Open reading frames were predicted with TransDecoder v2.0.1 [100] using the "-S" flag for paired end reads. Identified ORFs were aligned to PFAM (Release 31.0) with HMMer v3.1b2 [101] and to Swissprot (Release 2017_10) with BLAST v2.2.20. The resulting matches were used as input to TransDecoder together with the previously detected ORFs for a refined predictive round using the flags "--retain_pfam_hits" and "-retain_blastp_ hits".

\section{Differential expression analysis}

In each assembly, contigs containing multiple ORFs were split and replaced by the corresponding ORFs. The 
resulting sequences were then clustered with CD-HITest [102] using a $100 \%$ identity cutoff. This procedure removed redundancy while preserving as much as possible the assembled sequence diversity so as to reduce the risk of inducing misalignments during mapping. Reads from each semispecies were mapped to its corresponding reference using STAR v2.5.2b [103] with default parameters. Some of the mapping statistics produced by STAR are presented in Additional file 1, including the percentage of reads mapping to the assemblies and the percentage of mismatches per base.

Reads mapping to each contig were counted with FeatureCounts [104] using the flags "-M", "-s 1" and "-p" for counting multimapping reads, taking strand information into account, and counting fragments instead of reads, respectively. Contigs in the subsequent count table were clustered using CD-HIT with $98 \%$ identity cutoff at amino acid level for decreasing redundancy, removal of non-coding RNAs and reducing downstream issues with multiple testing. The resulting contigs and respective read counts were used as reference for the differential expression analysis. Contigs were not removed from the transcriptomes with basis on which organism they were associated with (Drosophila, Wolbachia, yeast or other bacteria) so as to avoid misalignments during mapping.

The differential expression analysis was done in $R$ v3.2.2 [105] with the DESeq2 v2_1.10.1 [106] package, which uses an inbuilt normalization pipeline. Tests were performed between WT and GFR samples of heads or abdomens of each sex. GFR was set as reference condition so that difference in expression could be read as being induced by Wolbachia. Contigs were considered differentially expressed if an adjusted pvalue (qvalue) $\leq$ 0.05 was observed in DESeq2's default Wald test. Contigs were said to be differentially expressed with high fold change whenever their absolute fold change value was greater than one standard deviation from the mean absolute fold change for the condition in which they were DE.

\section{Plots and statistics}

Principal component analyses were performed with DESeq2 and plotted with ggplot2 [107]. PCAs of individual semispecies were based on reads of that semispecies mapped to its own reference transcriptome, while PCAs of multiple semispecies are based on reads of all semispecies mapped to the OR reference transcriptome. Genes which contributed the most to each principal component (PC) were identified by their loading values. These were obtained by extracting the "rotation" element when calculating the PCA using the $\operatorname{prcomp}()$ function in R. Loadings were then plotted in ascending order and the genes whose values stood out in the beginning or end of the curve were selected. A chi-squared test was used to verify whether DE genes between GFR and WT flies were significantly overrepresented (pvalue < 0.05 ) among the genes which contributed the most to the separation between semispecies in the PCA plots.

Heatmaps of individual semispecies were generated with ggplot2 and were based on the expression values obtained in DESeq2 for the contigs DE between WT and GFR for that semispecies.

Venn diagrams were created in $\mathrm{R}$ using the VennDiagrams package [108] and are based on Drosophila genes identified as homologous between semispecies after clustering of the three differential expression reference transcriptomes with OrthoMCL [109] using default parameters. Venn diagram in Fig. 2 shows only Drosophila DE genes while the one in Additional file 2: Figure S1 includes all Drosophila genes - whether DE or not. BLAST searches to identify organisms associated to the genes associated to a single transcriptome in Additional file 2: Figure S1 were performed with protein BLAST against the non-redundant database of NCBI. Plots showing the number of DE genes in one or in multiple conditions were made in $\mathrm{R}$ with the upset $\mathrm{R}$ package [110].

\section{Contig and gene annotation}

DE contigs were annotated using two independent strategies. In the first one, all contigs were run through Interproscan v5.24-63.0 for GO term annotation.

In the second strategy, all contigs were blasted to a database containing genes from Drosophila willistoni, Drosophila melanogaster, Wolbachia, the yeast Saccharomyces cerevisiae and a number of Drosophila gut bacteria (Additional file 1). Contigs with a best hit to Wolbachia, other bacteria or yeast were discarded, while those with higher similarity to Drosophila were considered for annotation if the following criteria were met: the length of either the query or the subject, whichever was shortest, should correspond to at least $60 \%$ of the length of the longest, and the size of the aligned segment should correspond to at least $80 \%$ of the length of the shortest sequence.

Among the contigs which fulfilled these criteria, those with a best hit to $D$. melanogaster were directly annotated according to the Flybase D. melanogaster gff annotation file (release 6.18), while those with a best hit to $D$. willistoni had their annotation inferred from $D$. melanogaster orthologs listed in the Flybase gene ortholog table v2017_04. GO terms for each annotated contigs were extracted from the Flybase go-basic.obo file (release 201704-19). GO terms obtained through this method were generally considered more detailed than the ones annotated through InterproScan and were thus used for GO term enrichment analysis with the $\mathrm{R}$ package TopGo v2.22.0 [111]. TopGO's default "weight01" algorithm and 
Fisher's exact test statistic were employed in the analysis and GO terms were considered enriched when an adjusted pvalue $<0.05$ was obtained. In order to avoid biasing the GO enrichment analysis with eventual multiple copies of a same transcript, the analysis was performed on a dataset containing only one contig for each assembled "Trinity gene". This dataset was created using the script "extract_GO_assignments_from_Trinotate_ xls.pl", available with the Trinotate software package [112], which annotates each "Trinity gene" with the GO terms of all the contigs associated with it.

Contigs identified as differentially expressed were mapped against the KEGG (http://www.kegg.jp/) database for identification of metabolic pathways associated with them. The online tool blastKOALA (http://www. kegg.jp/blastkoala/) was used for this purposed, with Taxonomy ID set to 7215 (Drosophila) and KEGG gene database set to "family_eukaryotes + genus_prokaryotes". The metabolic map in Fig. 4 was prepared with the "search\&color pathway" function of the KEGG mapper tool (https://www.genome.jp/kegg/mapper.html) and redrawn in Adobe Illustrator.

\section{Additional files}

Additional file 1: Assembly metrics for the mapping reference transcriptomes of the AM, CA and OR semispecies. Transcriptome assembly metrics, including BUSCO marker recovery, ORF prediction and completeness, and the percentage of reads and ORFs associated with different organisms. (XLSX $24 \mathrm{~kb}$ )

Additional file 2: Additional figures. Figure S1. Overlap in Drosophila gene content between the transcriptomes of the three semispecies. Figure S2. Principal component analysis (a) and heatmap (b) of expression data of the AM semispecies. Figure S3. Principal component analysis (a) and heatmap (b) of expression data of the CA semispecies. Figure S4. Number of genes differentially expressed in one or multiple conditions of the AM semispecies. Figure S5. Number of genes differentially expressed in one or multiple conditions of the CA semispecies. Figure S6. First and second principal components in the PCA of female abdomen samples of all semispecies. Figure S7. Second and third principal components in the PCA of head samples of all semispecies mapped to the OR transcriptome. Figure S8. Principal component analysis of GFR head samples of all semispecies mapped to the OR transcriptome based on the same genes used in the WT head PCAs (Figs. 5c, 2d). Figure S9. Principal component analysis of abdomen samples of all semispecies mapped to the OR transcriptome. (PDF 2115 $\mathrm{kb})$

Additional file 3: All DE genes in the OR semispecies. Complete list of all DE genes in the OR semispecies including which condition, sex and tissue it was DE in, fold change, significance value, annotated Flybase gene identities for orthologs in D. willistoni and D. melanogaster, annotated gene name and domain predictions. (XLSX 505 kb)

Additional file 4: All DE genes in the CA semispecies. Complete list of all DE genes in the CA semispecies including which condition, sex and tissue it was DE in, fold change, significance value, annotated Flybase gene identities for orthologs in D. willistoni and D. melanogaster, annotated gene name and domain predictions. (XLSX $67 \mathrm{~kb}$ )

Additional file 5: All DE genes in the AM semispecies. Complete list of all DE genes in the AM semispecies including which condition, sex and tissue it was DE in, fold change, significance value, annotated Flybase gene identities for orthologs in D. willistoni and D. melanogaster, annotated gene name and domain predictions. (XLSX 69 kb)

Additional file 6: DE genes in multiple semispecies. List of all DE genes in more than one semispecies. (XLSX $17 \mathrm{~kb}$ )

Additional file 7: All enriched $\mathrm{GO}$ terms in the $\mathrm{AM}, \mathrm{CA}$ and $\mathrm{OR}$ semispecies. List of all GO terms enriched in each condition of the three semispecies. (XLSX $48 \mathrm{~kb}$ )

\section{Abbreviations}

AB: Andean-Brazilian; AM: Amazonian; AMP: Antimicrobial peptide; BCP: 1bromo-3-chloropropane; CA: Centro-American; CHC: Cuticle hydrocarbon; Cl: Cytoplasmic incompatibility; DE: Differentially expressed; GFR: Gut flora restored; GO: Gene ontology; IN: Interior; OBP: Odorant-binding protein; OR: Orinocan; ORF: Open reading frame; PC: Principal component; PCA: Principal component analysis; THF: Tetrahydrofolate; TR: Transitional; VAM: Ventral abdominal muscle; WT: Wild type

\section{Acknowledgements}

We would like to thank Johan Reimegård for running OrthoMCL, for advice and discussions regarding methods for RNA-sequencing and differential expression analysis, as well as for many insightful discussions on the obtained results. Sequencing was performed at the SNP\&SEQ Technology Platform in Uppsala, Sweden, which is part of the Swedish National Genomics Infrastructure.

\section{Authors' contributions}

LK and WJM designed the study. GB and LK performed bioinformatic analyses of the data. DSI extracted the RNA. GB and LK wrote the paper. All authors read and commented on the manuscript.

\section{Funding}

The project was funded by the Swedish research council VR grant 2014-4353 to LK, and by the Austrian Science Fund FWF grant P28255-B22 to WJM. The funding bodies had no role in the design of the study and collection, analysis, and interpretation of data or in writing the manuscript.

\section{Availability of data and materials}

The datasets generated and analyzed during the current study are available in the SRA database at NCBI, accession number PRJNA515416, https://www. ncbi.nlm.nih.gov/bioproject/PRJNA515416

Ethics approval and consent to participate

Not applicable

\section{Consent for publication}

Not applicable

\section{Competing interests}

The authors declare that they have no competing interests

\section{Author details}

${ }^{1}$ Molecular evolution, Department of Cell and Molecular Biology, Uppsala University, Husargatan 3, 75124 Uppsala, Sweden. ${ }^{2}$ Lab Genome Dynamics, Deparment Cell \& Developmental Biology, Center of Anatomy and Cell Biology, Medical University of Vienna, Schwarzspanierstraße 17, 1090 Vienna, Austria. ${ }^{3}$ Present address: Department of Epidemiology of Microbial Diseases, Yale University, 60 College Street, New Haven, CT 06510, USA.

Received: 4 January 2019 Accepted: 21 May 2019

Published online: 07 June 2019

\section{References}

1. Seehausen O, Butlin RK, Keller I, Wagner CE, Boughman JW, Hohenlohe PA, Peichel CL, Saetre G-P, Bank C, Brännström A, et al. Genomics and the origin of species. Nat Rev Genet. 2014;15:176.

2. Shropshire JD, Bordenstein SR. Speciation by Symbiosis: the Microbiome and Behavior. mBio. 2016;7(2):e01785.

3. Brucker RM, Bordenstein SR. Speciation by symbiosis. Trends Ecol Evol. 2012; 27(8):443-51. 
4. Dobzhansky T, Spassky B. Drosophila paulistorum, a cluster of species in statu nascendi. Proc Natl Academy Sci. 1959;45(3):419-28.

5. Ehrman L, Powell JR. The Drosophila willistoni species group. In: Ashburner M, Carson HL, Thompson Jr JN, editors. The Genetics and Biology of Drosophila, vol. 3b. New York: Academic Press Inc; 1982. p. 193-220.

6. Malogolowkin C. Races and Incipient Species in Drosophila paulistorum. Science. 1962;136(3513):327.

7. Ehrman L. Direct Observation of Sexual Isolation between Allopatric and between Sympatric Strains of the Different Drosophila paulistorum Races. Evolution. 1965:19(4):459-64.

8. Miller WJ, Ehrman L, Schneider D. Infectious speciation revisited: impact of symbiont-depletion on female fitness and mating behavior of Drosophila paulistorum. PLoS Pathog. 2010;6(12):e1001214.

9. Ehrman L. The genetics of hybrid sterility in Drosophila paulistorum. Evolution. 1960:14(2):212-23.

10. Dobzhansky T, Pavlovsky O. Experiments on the Incipient Species of the Drosophila paulistorum Complex. Genetics. 1967;55(1):141-56.

11. Ehrman L. Antibiotics and infectious hybrid sterility in Drosophila paulistorum. Mol Gen Genet MGG. 1968;103(3):218-22.

12. Kernaghan RP, Ehrman L. Antimycoplasmal Antibiotics and Hybrid Sterility in Drosophila paulistorum. Science. 1970;169(3940):63 LP-64.

13. Hilgenboecker $K$, Hammerstein $P$, Schlattmann $P$, Telschow A, Werren JH. How many species are infected with Wolbachia?--A statistical analysis of current data. FEMS Microbiol Lett. 2008;281(2):215-20.

14. Goodacre SL, Martin OY, Thomas CF, Hewitt GM. Wolbachia and other endosymbiont infections in spiders. Mol Ecol. 2006;15(2):517-27.

15. Cordaux R, Pichon S, Hatira HB, Doublet V, Greve P, Marcade I, BraquartVarnier C, Souty-Grosset C, Charfi-Cheikhrouha F, Bouchon D. Widespread Wolbachia infection in terrestrial isopods and other crustaceans. ZooKeys. 2012;176:123-31.

16. Bandi C, Anderson TJ, Genchi C, Blaxter ML. Phylogeny of Wolbachia in filarial nematodes. Proc Biol Sci. 1998;265(1413):2407-13.

17. Strunov A, Schneider DI, Albertson R, Miller WJ. Restricted distribution and lateralization of mutualistic Wolbachia in the Drosophila brain. Cell Microbiol. 2017;19(1):e12639.

18. Pietri JE, DeBruhl $H$, Sullivan W. The rich somatic life of Wolbachia. MicrobiologyOpen. 2016;5(6):923-36.

19. Zug R, Hammerstein P. Bad guys turned nice? A critical assessment of Wolbachia mutualisms in arthropod hosts. Biol Rev Camb Philos Soc. 2015; 90(1):89-111.

20. Hosokawa T, Koga R, Kikuchi Y, Meng XY, Fukatsu T. Wolbachia as a bacteriocyte-associated nutritional mutualist. Proc Natl Acad Sci U S A. 2010; 107(2):769-74.

21. Dedeine F, Bouletreau M, Vavre F. Wolbachia requirement for oogenesis: occurrence within the genus Asobara (Hymenoptera, Braconidae) and evidence for intraspecific variation in A. tabida. Heredity. 2005;95(5):394-400.

22. Dedeine F, Vavre F, Fleury F, Loppin B, Hochberg ME, Bouletreau M. Removing symbiotic Wolbachia bacteria specifically inhibits oogenesis in a parasitic wasp. Proc Natl Acad Sci U S A. 2001;98(11):6247-52.

23. Schneider DI, Ehrman L, Engl T, Kaltenpoth M, Hua-Van A, Le Rouzic A, Miller WJ. Symbiont-Driven Male Mating Success in the Neotropical Drosophila paulistorum Superspecies. Behavior Genetics. 2018;49(1):83-98.

24. Larter NK, Sun JS, Carlson JR. Organization and function of Drosophila odorant-binding proteins. Elife. 2016;5:e20242.

25. Swarup S, Williams IT, Anholt HRR. Functional dissection of Odorant-binding protein genes in Drosophila melanogaster. Genes Brain Behav. 2011;10:64857.

26. Grillet M, Dartevelle L, Ferveur JF. A Drosophila male pheromone affects female sexual receptivity. Proc Biol Sci. 2006;273(1584):315-23.

27. Chung H, Carroll SB. Wax, sex and the origin of species: Dual roles of insect cuticular hydrocarbons in adaptation and mating. Bioessays. 2015;37(7):82230.

28. Chao TH, Ehrman L, Permaul A, Vincent R, Sattaur L, Brandt D. Male-specific cuticular compounds of the six Drosophila paulistorum semispecies: structural identification and mating effect. J Chem Ecol. 2010;36(9):933-42.

29. Wade MJ. Infectious speciation. Nature. 2001:409(6821):675-7.

30. Bordenstein SR, O'Hara FP, Werren JH. Wolbachia-induced incompatibility precedes other hybrid incompatibilities in Nasonia. Nature. 2001;409(6821): 707-10.

31. Beckmann JF, Ronau JA, Hochstrasser M. A Wolbachia deubiquitylating enzyme induces cytoplasmic incompatibility. Nat Microbiol. 2017;2:17007.
32. Serbus LR, Casper-Lindley C, Landmann F, Sullivan W. The genetics and cell biology of Wolbachia-host interactions. Annu Rev Genet. 2008;42:683-707.

33. LePage DP, Metcalf JA, Bordenstein SR, On J, Perlmutter JI, Shropshire JD, Layton EM, Funkhouser-Jones LJ, Beckmann JF, Bordenstein SR. Prophage WO genes recapitulate and enhance Wolbachia-induced cytoplasmic incompatibility. Nature. 2017;543(7644):243-7.

34. Grote A, Voronin D, Ding T, Twaddle A, Unnasch TR, Lustigman S, Ghedin E. Defining Brugia malayi and Wolbachia symbiosis by stage-specific dual RNAseq. PLoS Negl Trop Dis. 2017;11(3):e0005357.

35. Wu M, Sun LV, Vamathevan J, Riegler M, Deboy R, Brownlie JC, McGraw EA, Martin W, Esser C, Ahmadinejad N, et al. Phylogenomics of the reproductive parasite Wolbachia pipientis wMel: a streamlined genome overrun by mobile genetic elements. PLoS Biol. 2004;2(3):E69.

36. Kohyama-Koganeya A, Kim Y-J, Miura M, Hirabayashi Y. A Drosophila orphan G protein-coupled receptor BOSS functions as a glucose-responding receptor: Loss of boss causes abnormal energy metabolism. Proc Natl Acad Sci. 2008;105(40):15328.

37. Reichhart JM, Gubb D, Leclerc V. Chapter eleven - The Drosophila Serpins: Multiple Functions in Immunity and Morphogenesis. In: Methods in Enzymology. Edited by Whisstock JC, Bird PI. San Diego: Academic Press. 2011:499:205-25.

38. Yi H-Y, Chowdhury M, Huang Y-D, Yu X-Q. Insect antimicrobial peptides and their applications. Appl Microbiol Biotechnol. 2014;98(13):5807-22.

39. Pal S, Wu J, Wu LP. Microarray analyses reveal distinct roles for Rel proteins in the Drosophila immune response. Dev Comp Immunol. 2008;32(1):50-60.

40. Vanha-aho L-M, Anderl I, Vesala L, Hultmark D, Valanne S, Rämet M. Edin Expression in the Fat Body Is Required in the Defense Against Parasitic Wasps in Drosophila melanogaster. PLOS Pathogens. 2015;11(5):e1004895.

41. Chertemps T, Younus F, Steiner C, Durand N, Coppin CW, Pandey G, Oakeshott JG, Maïbèche M. An antennal carboxylesterase from Drosophila melanogaster, esterase 6 , is a candidate odorant-degrading enzyme toward food odorants. Front Physiol. 2015:6:315.

42. Min K-T, Benzer S. Preventing Neurodegeneration in the Drosophila Mutant bubblegum. Science. 1999;284(5422):1985.

43. Burmester T, Antoniewski C, Lepesant JA. Ecdysone-regulation of synthesis and processing of Fat Body Protein 1, the larval serum protein receptor of Drosophila melanogaster. Eur J Biochem. 2001;262(1):49-55.

44. Von Stetina JR, Orr-Weaver TL. Developmental Control of Oocyte Maturation and Egg Activation in Metazoan Models. Cold Spring Harbor Perspect Biol. 2011;3(10):a005553.

45. Avilés-Pagán EE, Orr-Weaver TL. Activating embryonic development in Drosophila. Semin Cell Dev Biol. 2018;84:100-10.

46. Robertson SE, Dockendorff TC, Leatherman JL, Faulkner DL, Jongens TA. germ cell-less Is Required Only during the Establishment of the Germ Cell Lineage of Drosophila and Has Activities Which Are Dependent and Independent of Its Localization to the Nuclear Envelope. Dev Biol. 1999; 215(2):288-97.

47. Styhler S, Nakamura A, Lasko P. VASA localization requires the SPRY-domain and SOCS-box containing protein, GUSTAVUS. Dev Cell. 2002;3(6):865-76.

48. Findlay GD, Sitnik JL, Wang W, Aquadro CF, Clark NL, Wolfner MF. Evolutionary Rate Covariation Identifies New Members of a Protein Network Required for Drosophila melanogaster Female Postmating Responses. PLOS Genetics. 2014;10(1):e1004108.

49. Xi Z, Gavotte L, Xie Y, Dobson SL. Genome-wide analysis of the interaction between the endosymbiotic bacterium Wolbachia and its Drosophila host. BMC Genomics. 2008;9:1.

50. Lazareva AA, Roman G, Mattox W, Hardin PE, Dauwalder B. A Role for the Adult Fat Body in Drosophila Male Courtship Behavior. PLOS Genetics. 2007; 3(1):e16.

51. Chung H, Sztal T, Pasricha S, Sridhar M, Batterham P, Daborn PJ. Characterization of Drosophila melanogaster cytochrome P450 genes. Proc Natl Acad Sci USA. 2009;106(14):5731-6.

52. Hughes GL, Ren X, Ramirez JL, Sakamoto JM, Bailey JA, Jedlicka AE, Rasgon JL. Wolbachia Infections in Anopheles gambiae Cells: Transcriptomic Characterization of a Novel Host-Symbiont Interaction. PLOS Pathogens. 2011;7(2):e1001296.

53. Zheng Y, Wang J-L, Liu C, Wang C-P, Walker T, Wang Y-F. Differentially expressed profiles in the larval testes of Wolbachia infected and uninfected Drosophila. BMC Genomics. 2011;12(1):595.

54. Caragata EP, Pais FS, Baton LA, Silva JB, Sorgine MH, Moreira LA. The transcriptome of the mosquito Aedes fluviatilis (Diptera: Culicidae), and 
transcriptional changes associated with its native Wolbachia infection. BMC Genomics. 2017;18(1):6.

55. Grobler Y, Yun CY, Kahler DJ, Bergman CM, Lee H, Oliver B, Lehmann R. Whole genome screen reveals a novel relationship between Wolbachia levels and Drosophila host translation. PLoS Pathog. 2018;14(11):e1007445.

56. Matsuoka S, Armstrong AR, Sampson LL, Laws KM, Drummond-Barbosa D. Adipocyte Metabolic Pathways Regulated by Diet Control the Female Germline Stem Cell Lineage in Drosophila melanogaster. Genetics. 2017; 206(2):953-71.

57. Sonn JY, Lee J, Sung MK, Ri H, Choi JK, Lim C, Choe J. Serine metabolism in the brain regulates starvation-induced sleep suppression in Drosophila melanogaster. Proc Natl Acad Sci. 2018.

58. Morioka E, Oida M, Tsuchida T, Ikeda M. Nighttime activities and peripheral clock oscillations depend on Wolbachia endosymbionts in flies. Scientific Reports. 2018;8(1):15432.

59. Caragata EP, Rancès E, Hedges LM, Gofton AW, Johnson KN, O'Neill SL, McGraw EA. Dietary Cholesterol Modulates Pathogen Blocking by Wolbachia. PLoS Pathogens. 2013;9(6):e1003459.

60. Geoghegan V, Stainton K, Rainey SM, Ant TH, Dowle AA, Larson T, Hester S, Charles PD, Thomas B, Sinkins SP. Perturbed cholesterol and vesicular trafficking associated with dengue blocking in Wolbachia-infected Aedes aegypti cells. Nat Commun. 2017:8:526.

61. Peters J, Byrne Gl. Chlamydia trachomatis growth depends on eukaryotic cholesterol esterification and is affected by Acyl-CoA:cholesterol acyltransferase inhibition. Pathog Dis. 2015;73(6):ftv028.

62. Tirmarche S, Kimura S, Dubruille R, Horard B, Loppin B. Unlocking sperm chromatin at fertilization requires a dedicated egg thioredoxin in Drosophila. Nature Commun. 2016;7:13539.

63. Weiss BL, Wang J, Aksoy S. Tsetse Immune System Maturation Requires the Presence of Obligate Symbionts in Larvae. PLoS Biology. 2011;9(5): e1000619.

64. Weiss BL, Maltz M, Aksoy S. Obligate symbionts activate immune system development in the tsetse fly. J Immunol. 2012;188(7):3395-403.

65. Ye YH, Woolfit M, Rancès E, O'Neill SL, McGraw EA. Wolbachia-Associated Bacterial Protection in the Mosquito Aedes aegypti. PLOS Negl Trop Dis. 2013;7(8):e2362.

66. Wong ZS, Hedges LM, Brownlie JC, Johnson KN. Wolbachia-Mediated Antibacterial Protection and Immune Gene Regulation in Drosophila. PLOS ONE. 2011;6(9):e25430.

67. Teixeira L, Ferreira A, Ashburner M. The bacterial symbiont Wolbachia induces resistance to RNA viral infections in Drosophila melanogaster. PLoS Biol. 2008;6(12):e2.

68. Hedges LM, Brownlie JC, O'Neill SL, Johnson KN. Wolbachia and virus protection in insects. Science. 2008;322(5902):702.

69. Eleftherianos I, Atri J, Accetta J, Castillo J. Endosymbiotic bacteria in insects: guardians of the immune system? Front Physiol. 2013;4:46.

70. Gupta V, Vasanthakrishnan RB, Siva-Jothy J, Monteith KM, Brown SP, Vale PF. The route of infection determines Wolbachia antibacterial protection in Drosophila. Proc R Soc B Biol Sci. 2017;284(1856):20170809.

71. Login FH, Balmand S, Vallier A, Vincent-Monégat C, Vigneron A, Weiss-Gayet M, Rochat D, Heddi A. Antimicrobial Peptides Keep Insect Endosymbionts Under Control. Science. 2011;334(6054):362.

72. Barajas-Azpeleta R, Wu J, Gill J, Welte R, Seidel C, McKinney S, Dissel S, Si K. Antimicrobial peptides modulate long-term memory. PLoS Genet. 2018; 14(10):e1007440

73. Thomas P, Kenny N, Eyles D, Moreira LA, O'Neill SL, Asgari S. Infection with the wMel and wMelPop strains of Wolbachia leads to higher levels of melanization in the hemolymph of Drosophila melanogaster, Drosophila simulans and Aedes aegypti. Dev Comp Immunol. 2011;35(3):360-5.

74. Christensen S, Perez Dulzaides R, Hedrick VE, Momtaz AJ, Nakayasu ES, Paul LN, Serbus LR. Wolbachia Endosymbionts Modify Drosophila Ovary Protein Levels in a Context-Dependent Manner. Appl Environ Microbiol. 2016;82(17): 5354-63.

75. Mazor KM, Dong L, Mao Y, Swanda RV, Qian S-B, Stipanuk MH. Effects of single amino acid deficiency on mRNA translation are markedly different for methionine versus leucine. Scientific Reports. 2018;8(1):8076.

76. Evans O, Caragata EP, McMeniman CJ, Woolfit M, Green DC, Williams CR, Franklin CE, O'Neill SL, McGraw EA. Increased locomotor activity and metabolism of Aedes aegypti infected with a life-shortening strain of Wolbachia pipientis. J Exp Biol. 2009;212(Pt 10:1436-41.
77. Taghli-Lamallem O, Plantie E, Jagla K. Drosophila in the Heart of Understanding Cardiac Diseases: Modeling Channelopathies and Cardiomyopathies in the Fruitfly. J Cardiovasc Dev Dis. 2016;3(1):7.

78. Pons M, Soulard C, Soustelle L, Parmentier M-L, Grau Y, Layalle S. A New Behavioral Test and Associated Genetic Tools Highlight the Function of Ventral Abdominal Muscles in Adult Drosophila. Front Cell Neurosci. 2017;11:371.

79. Trott AR, Donelson NC, Griffith LC, Ejima A. Song Choice Is Modulated by Female Movement in Drosophila Males. PLoS ONE. 2012;7(9):e46025.

80. Yu Y, Huang R, Ye J, Zhang V, Wu C, Cheng G, Jia J, Wang L. Regulation of starvation-induced hyperactivity by insulin and glucagon signaling in adult Drosophila. eLife. 2016;5:e15693.

81. Ganassi S, Tagliazucchi D, Mola L. Occurrence of nitric oxide synthase in Megoura viciae Buckton (Homoptera, Aphididae): an histochemical and immunohistochemical localisation. Eur J Histochem. 2005;49(4):385-93.

82. Chung H, Loehlin DW, Dufour HD, Vaccarro K, Millar JG, Carroll SB. A Single Gene Affects Both Ecological Divergence and Mate Choice in Drosophila. Science. 2014;343(6175):1148.

83. Grillet M, Everaerts C, Houot B, Ritchie MG, Cobb M, Ferveur J-F. Incipient speciation in Drosophila melanogaster involves chemical signals. Sci Reports. 2012;2:224.

84. Marcillac F, Bousquet F, Alabouvette J, Savarit F, Ferveur J-F. A Mutation With Major Effects on Drosophila melanogaster Sex Pheromones. Genetics. 2005;171(4):1617-28

85. Xu P, Atkinson R, Jones DN, Smith DP. Drosophila OBP LUSH is required for activity of pheromone-sensitive neurons. Neuron. 2005;45(2):193-200.

86. Billeter JC, Levine J. The role of CVA and the Odorant binding protein Lush in social and sexual behavior in Drosophila melanogaster. Front Ecol Evol. 2015;3:75.

87. Chertemps T, François A, Durand N, Rosell G, Dekker T, Lucas P, MaïbècheCoisne M. A carboxylesterase, Esterase-6, modulates sensory physiological and behavioral response dynamics to pheromone in Drosophila. BMC Biology. 2012;10(1):56.

88. WGv N. Drosophila Pheromones: From Reception to Perception. In: Mucignat-Caretta C, editor. Neurobiology of Chemical Communication. Boca Raton: CRC Press/Taylor \& Francis; 2014

89. Greenspan RJ, Ferveur J-F. Courtship in Drosophila. Annu Rev Genetics. 2000; 34(1):205-32.

90. Majewska MM, Suszczynska A, Kotwica-Rolinska J, Czerwik T, Paterczyk B, Polanska MA, Bernatowicz P, Bebas P. Yolk proteins in the male reproductive system of the fruit fly Drosophila melanogaster: Spatial and temporal patterns of expression. Insect Biochem Mol Biol. 2014;47:23-35.

91. Tarone AM, Mclntyre LM, Harshman LG, Nuzhdin SV. Genetic variation in the Yolk protein expression network of Drosophila melanogaster: sex-biased negative correlations with longevity. Heredity. 2012;109:226.

92. Garabedian MJ, Hung MC, Wensink PC. Independent control elements that determine yolk protein gene expression in alternative Drosophila tissues. Proc Natl Acad Sci. 1985;82(5):1396.

93. Arbuthnott D, Fedina TY, Pletcher SD, Promislow DEL. Mate choice in fruit flies is rational and adaptive. Nat Commun. 2017;8:13953.

94. Veen T, Borge T, Griffith SC, Saetre G-P, Bures S, Gustafsson L, Sheldon BC. Hybridization and adaptive mate choice in flycatchers. Nature. 2001;411:45.

95. Andrews S. FastQC: a quality control tool for high throughput sequence data. 2010. Available online at: http://www.bioinformatics.babraham.ac.uk/ projects/fastqc/.

96. Bolger AM, Lohse M, Usadel B. Trimmomatic: a flexible trimmer for Illumina sequence data. Bioinformatics. 2014;30(15):2114-20.

97. Grabherr MG, Haas BJ, Yassour M, Levin JZ, Thompson DA, Amit I, Adiconis X, Fan L, Raychowdhury R, Zeng Q, et al. Full-length transcriptome assembly from RNA-Seq data without a reference genome. Nat Biotechnol. 2011;29(7):644-52.

98. Simao FA, Waterhouse RM, loannidis P, Kriventseva EV, Zdobnov EM. BUSCO: assessing genome assembly and annotation completeness with single-copy orthologs. Bioinformatics. 2015;31(19):3210-2.

99. Li H, Durbin R. Fast and accurate short read alignment with BurrowsWheeler transform. Bioinformatics. 2009;25(14):1754-60.

100. Haas BJ, Papanicolaou A, Yassour M, Grabherr M, Blood PD, Bowden J, Couger MB, Eccles D, Li B, Lieber M, et al. De novo transcript sequence reconstruction from RNA-seq using the Trinity platform for reference generation and analysis. Nature Protocols. 2013;8(8):1494-512.

101. Eddy SR, Wheeler TJ. team Hd: HMMER: biosequence analysis using profile hidden Markov models. 2015. Available online at: http://hmmerorg/. 
102. Fu L, Niu B, Zhu Z, Wu S, Li W. CD-HIT: accelerated for clustering the nextgeneration sequencing data. Bioinformatics. 2012;28(23):3150-2.

103. Dobin A, Davis CA, Schlesinger F, Drenkow J, Zaleski C, Jha S, Batut $P$, Chaisson M, Gingeras TR. STAR: ultrafast universal RNA-seq aligner. Bioinformatics. 2013;29(1):15-21.

104. Liao Y, Smyth GK, Shi W. FeatureCounts: an efficient general purpose program for assigning sequence reads to genomic features. Bioinformatics. 2014;30(7):923-30.

105. R-team. R: A Language and Environment for Statistical Computing. 2015. Available online at: https://wwwR-projectorg.

106. Love Ml, Huber W, Anders S. Moderated estimation of fold change and dispersion for RNA-seq data with DESeq2. Genome Biol Evol. 2014;15:550.

107. Wickham H. Ggplot2: Elegant Graphics for Data Analysis. New York: Springer-Verlag; 2016.

108. Chen H, Boutros PC. VennDiagram: a package for the generation of highlycustomizable Venn and Euler diagrams in R. BMC Bioinformatics. 2011;12(1):35.

109. Li L, Stoeckert CJ, Roos DS. OrthoMCL: Identification of Ortholog Groups for Eukaryotic Genomes. Genome Res. 2003;13(9):2178-89.

110. Lex A, Gehlenborg N, Strobelt H, Vuillemot R, Pfister H. UpSet: Visualization of Intersecting Sets. IEEE Trans Vis Comput Graph. 2014;20(12):1983-92.

111. Alexa A, Rahnenfuhrer J: TopGO: Enrichment Analysis for Gene Ontology. In. 2.30 .1 edn; 2016.

112. Bryant DM, Johnson K, DiTommaso T, Tickle T, Couger MB, Payzin-Dogru D, Lee TJ, Leigh ND, Kuo T-H, Davis FG, et al. A Tissue-Mapped Axolotl De Novo Transcriptome Enables Identification of Limb Regeneration Factors. Cell Reports. 2017;18(3):762-76.

\section{Publisher's Note}

Springer Nature remains neutral with regard to jurisdictional claims in published maps and institutional affiliations.

Ready to submit your research? Choose BMC and benefit from:

- fast, convenient online submission

- thorough peer review by experienced researchers in your field

- rapid publication on acceptance

- support for research data, including large and complex data types

- gold Open Access which fosters wider collaboration and increased citations

- maximum visibility for your research: over $100 \mathrm{M}$ website views per year

At $\mathrm{BMC}$, research is always in progress.

Learn more biomedcentral.com/submissions 